\author{
Universidade de São Paulo \\ Instituto de Física
}

\title{
Ensemble Beta-Laguerre pseudo-hermitiano
}

\author{
Cleverson Andrade Goulart
}

Orientador: Prof. Dr. Mauricio Porto Pato

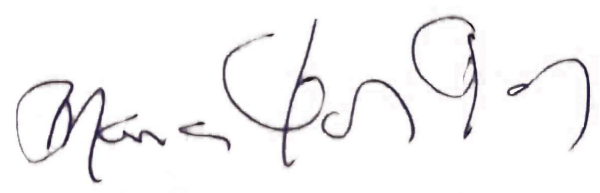

Dissertação de mestrado apresentada ao Instituto de Física como requisito parcial para a obtenção do título de Mestre em Ciências.

Banca Examinadora:

Prof. Dr. Mauricio Porto Pato - IF-USP

Prof. Dr. José Fernando Fontanari - IFSC-USP

Prof. Dr. Edson Denis Leonel - UNESP 


\section{FICHA CATALOGRÁFICA \\ Preparada pelo Serviço de Biblioteca e Informação do Instituto de Física da Universidade de São Paulo}

Goulart, Cleverson Andrade

Ensemble Beta-Laguerre pseudo-hermitiano. São Paulo, 2021.

Dissertação (Mestrado) - Universidade de São Paulo, Instituto de Física, Depto. de Física Matemática

Orientador: Prof. Dr. Mauricio Porto Pato

Área de Concentração: Física

Unitermos: 1. Mecânica quântica pseudo-hermitiana; 2. Teoria de matrizes aleatórias; 3. $\beta$ (Beta)-Laguerre ensemble; 4. Estatística de níveis.

USP/IF/SBI-026/2021 


\title{
University of São Paulo \\ Physics Institute
}

\section{Ensemble Beta Laguerre Pseudo-Hermitian}

\author{
Cleverson Andrade Goulart
}

Supervisor: Prof. Dr. Mauricio Porto Pato

Dissertation submitted to the Physics Institute of the University of São Paulo in partial fulfillment of the requirements for the degree of Master of Science.

Examining Committee:

Prof. Dr. Mauricio Porto Pato - IF-USP

Prof. Dr. José Fernando Fontanari - IFSC-USP

Prof. Dr. Edson Denis Leonel - UNESP 

À Clarinda da Silva Goulart e Fátima Goulart pelo apoio e lições que me fortaleceram, muito, ao longo deste processo. 


\section{Agradecimentos}

Aos meus pais, familiares e amigos pelo apoio e compreensão. Um agradecimento em especial para minhas amigas Flávia e Gisele, ichis, que sempre incentivaram e estiveram por perto durante todo o meu processo acadêmico, vocês são incríveis! À minha Tia Isabel pela ajuda na revisão ortográfica do texto, suas dicas foram, sem dúvida, úteis. Ao Gabriel Marinello, pelas conversas e dicas que ajudaram a deixar o texto mais claro e preciso. Aproveito e agradeço, também:

- Ao meu orientador Prof. Dr. Mauricio Porto Pato, pela confiança e aprendizado na realização do trabalho.

- À Universidade de São Paulo e em especial a comunidade do Instituto de Física, professores e colegas, pelo valioso aprendizado nessa bela ciência.

- Ao conselho Nacional de Desenvolvimento Científico e Tecnológico (CNPq), pelo apoio financeiro (Processo 133394/2019-0). 


\section{Resumo}

$\mathrm{Na}$ década de 30, as matrizes aleatórias (RM) foram introduzidas pelo matemático John Wishart em um estudo estatístico de sistemas multivariados. Algumas décadas depois a primeira aplicação em física aconteceu no estudo dos níveis energéticos de núcleos atômicos pesados com Eugene P. Wigner. Com o desenvolvimento de trabalhos recentes, construindo matrizes aleatórias tridiagonais conhecidas como beta-ensembles, investigou-se o efeito do índice beta no contínuo, ou seja, fora do threefold way de Dyson, a saber $\beta=1,2,4$. Motivados por buscar no contexto das matrizes aleatórias elementos com a mesma propriedade de hamiltonianas não hermitianas, mas invariantes, simultaneamente, por simetrias de paridade $(\mathrm{P})$ e inversão temporal $(\mathrm{T})$ estudamos, neste trabalho, a estatística de sistemas pseudohermitianos no ensemble Beta-Laguerre. Essa classe de elementos compartilham o espectro com o seu respectivo adjunto via transformação de similaridade. Os resultados obtidos mostram a concordância entre as expressões analíticas, revisadas ao longo do estudo desenvolvido, com o perfil de histogramas produzidos empregando cálculo numérico para diferentes parâmetros em matrizes no ensemble utilizado. Destaca-se o resultado observado na estatística de espaçamento de autovalores, que difere entre os casos hermitianos e pseudo-hermitianos. Esse fenômeno, que também é observado no ensemble Beta-Hermite, indica que embora a distribuição dos elementos pseudo-hermitanos tenda, no limite assintótico, a distribuição dos elementos hermitianos, o parâmetro $\beta$ que ajusta as expressões difere, concluímos que a relação é $\beta_{\text {psudo-hermitiano }}=2 \cdot \beta_{\text {hermitiano }}$.

Palavras-chave: Mecânica quântica pseudo-hermitiana; Teoria de matrizes aleatórias; $\beta$-Laguerre ensemble; Estatística de níveis; 


\section{Abstract}

Random matrices (RM) were introduced by the mathematician John Wishart in the 1930s in a statistical study of multivariate systems. A few decades later the first application in physics took place in the study of the energy levels of heavy atomic nuclei in Wigner's work. With the development of recent works, building random tridiagonal matrices knows as beta-ensembles, the effect of the beta index on the continuum, out of the Dyson's threefold Way $\beta=1,2,4$, was investigated. Motivated by searching in the context of random matrices for elements with the same property as non-Hermitian Hamiltonians that are invariants under the combined symmetries of parity $(\mathrm{P})$ and temporal reversion $(\mathrm{T})$, we study in this work the statistic of pseudo-Hermitian systems in the Beta-Laguerre ensemble. This class of elements shares the spectrum with its respective adjoint by a similarity transformation. The results obtained show an agreement between the analytical expressions, revised throughout the developed study, with the profile of histograms obtained using numerical calculation for different parameters in matrices in the ensemble used. We highlight the result observed in the eigenvalue spacing statistics, which differs between the Hermitian and pseudohermitian cases. This phenomenon, which is also observed in the BetaHermite ensemble, indicates that although the distribution of the pseudo-Hermitan elements tends, in the asymptotic limit, the distribution of the Hermitian elements, the parameter that fit the expressions differs, we conclude that the relationship is $\beta_{\text {pseudo-hermitiano }}=2 \cdot \beta_{\text {hermitiano }}$.

Keywords: Pseudo-Hermitian quantum mechanics; Randon matrix theory; $\beta$-Laguerre ensemble; Level statistics. 


\section{Conteúdo}

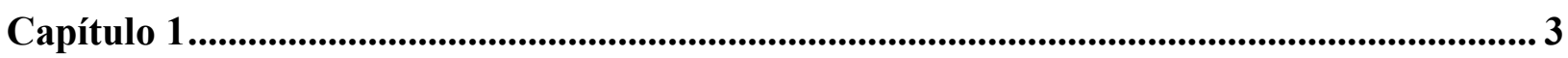

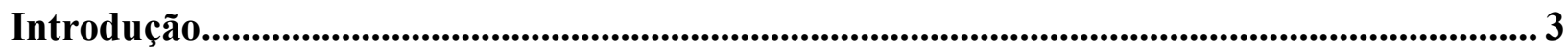

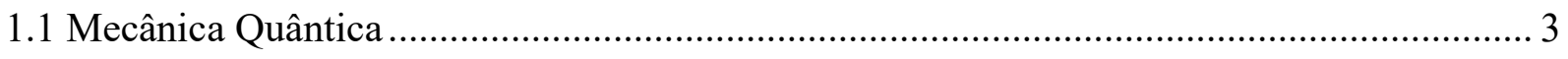

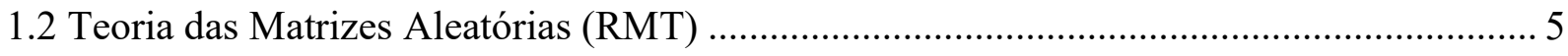

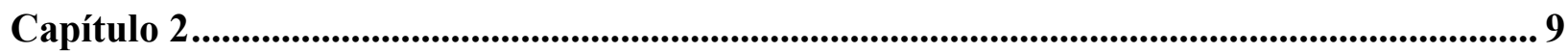

Ensemble de Wishart ..................................................................................................................... 9

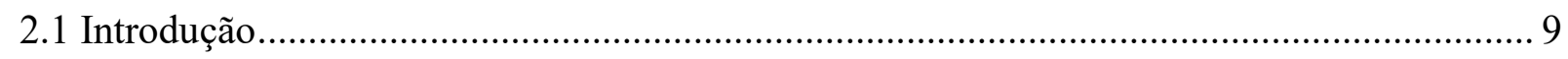

2.2 Densidade de probabilidade conjunta dos elementos de entrada ................................ 10

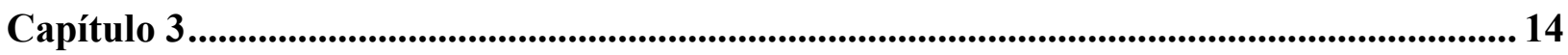

3. Estatística dos Autovalores .................................................................................................... 15

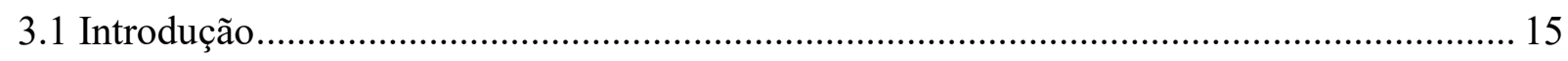

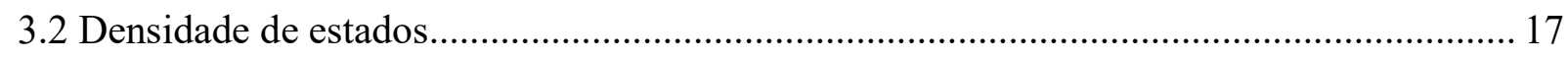

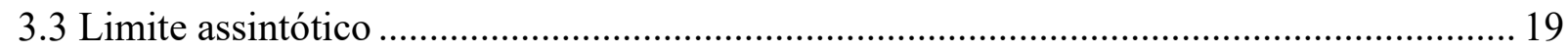

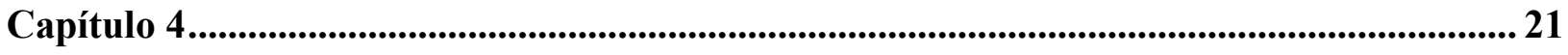

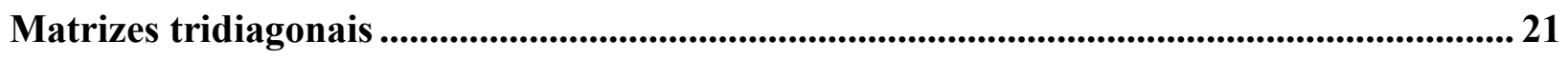

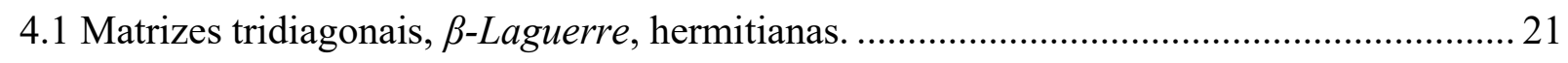

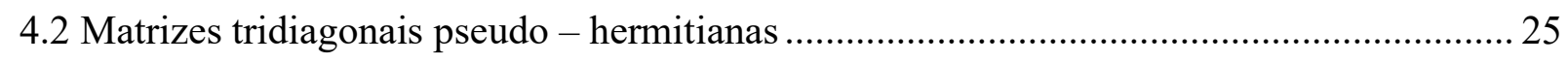

Capítulo 5

Resultados Numéricos.................................................................................................................... 30

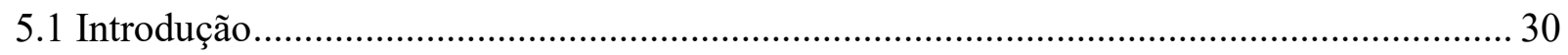

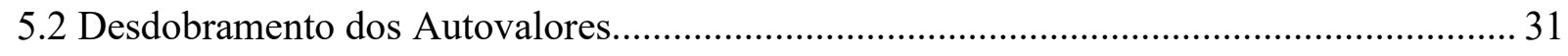

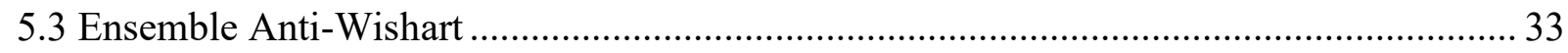




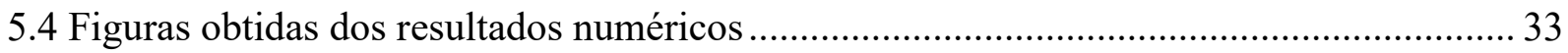

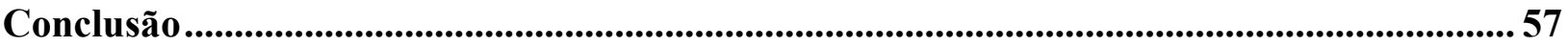

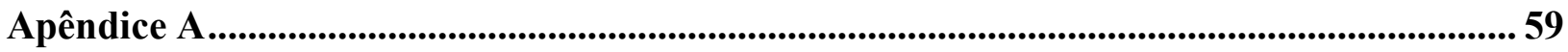

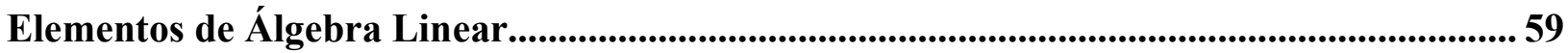

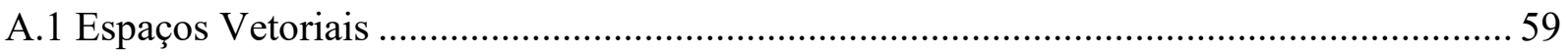

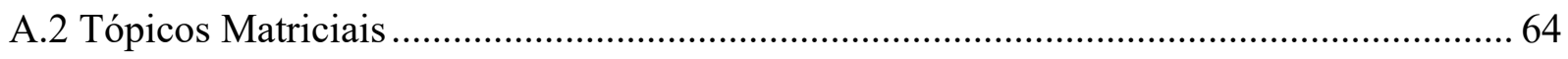

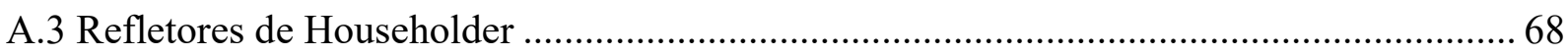

A.4 Determinante de Vandermonde............................................................................ 71

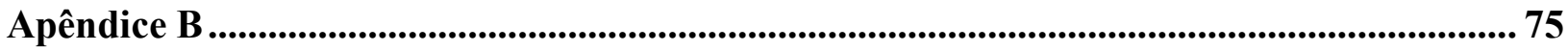

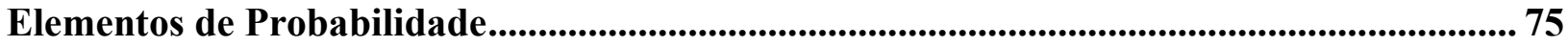

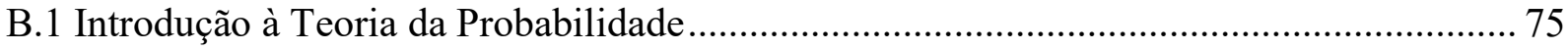

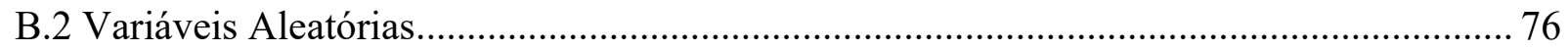

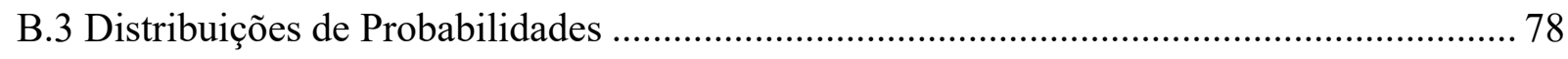

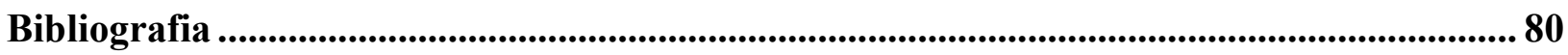




\section{Capítulo 1}

\section{Introdução}

Neste capítulo são introduzidas algumas ideias importantes da mecânica quântica que facilitam a motivação no uso de matrizes aleatórias para o estudo de certos sistemas físicos. Em seguida, passamos para a definição de matrizes aleatórias assim como uma classificação que é feita devido as simetrias de um dado ensemble. Completando a discussão é apresentada uma estrutura da organização desta dissertação.

\subsection{Mecânica Quântica}

No século XX, a física foi marcada pelo desenvolvimento da chamada teoria quântica. Essa teoria fornece a descrição para sistemas microscópicos (sistemas em que a ação $S$ possui ordem de grandeza da constante de Planck $(S \sim h)$. A mecânica quântica baseia-se em duas estruturas: vetores no espaço de Hilbert $|\Psi\rangle$ e operadores $\widehat{O}[2]$. A primeira estrutura contém toda a informação acerca de um sistema, enquanto a segunda é um instrumento matemático, da teoria, utilizado para acessar essas informações. Nesta teoria, a evolução dinâmica de um sistema pode ser obtida através de uma equação de autovalor e autovetor conhecida como equação de Schrödinger [1,2]:

$$
\hat{H}|\Psi(t)\rangle=E|\Psi(t)\rangle
$$

sendo $\hat{H}$ um operador hermitiano denominado Hamiltoniano, e $|\Psi(t)\rangle$ um vetor de estado, ambos no espaço vetorial de Hilbert. Observamos pela equação acima que a aplicação do operador Hamiltoniano sobre um vetor de estado produz um espectro que pode ser contínuo ou discreto. Esse espectro corresponde aos autovalores $E$ do operador, e nesse caso representam as energias possíveis de serem acessadas por um sistema. Essa equação nos ajuda a entender o problema matemático central da teoria 
quântica matricial. Dada uma matriz que representa um operador, busca-se os respectivos autovalores (espectro) via equação (1), por exemplo. Uma classe de operadores que desempenham um papel importante nessa teoria são os chamados operadores hermitianos. Considere que $V$ seja um espaço vetorial sobre um corpo $F$ e as seguintes definições ${ }^{1}$.

Definição 1.1: Uma transformação linear $T: V \rightarrow V$ é denominado operador linear.

Uma vantagem de trabalhar com operadores lineares é que podemos representá-los por uma matriz de dimensão correspondente aquela do espaço vetorial estudado.

Definição 1.2: Dado um operador linear $T$, que em uma determinada base possui representação matricial da forma $T_{i j}$, podemos definir a matriz que representa o operador adjunto $T^{\dagger}$ como o transposto conjugado de $T$, ou seja, $\left(T_{i j}\right)^{\dagger}=T_{j i}^{*}$.

Com as definições acima, estamos agora em condições de definir um operador hermitiano.

Definição 1.3: Um operador $O$ é dito hermitano se ele corresponder ao seu adjunto.

$$
O=O^{\dagger}
$$

O espectro de operadores hermitianos são números reais, o que nos leva imediatamente que um observável deve ser representado por essa classe. No final da década de 90 Bender [3] observou que hamiltonianos não hermitianos, mas invariantes por simetrias combinadas de paridade $P$ e inversão temporal $T$, apresentavam espectro real. Os primeiros hamiltonianos, pseudo-hermitianos, estudados eram da forma

$$
\hat{H}=(\hat{p})+(\hat{x})^{2}-(i x)^{n}, n \in \mathbb{R}
$$

Esse estudo, mostrava a necessidade de estender a mecânica quântica a fim de incluir sistemas que possuíam hamiltonianas com as referidas simetrias. De um ponto de vista mais geral, a propriedade

\footnotetext{
${ }^{1}$ Uma discussão mais detalhada sobre álgebra linear é apresentada no apêndice A.
} 
determinante dessa classe de operadores é que eles compartilham o mesmo espectro com seus adjuntos [4].

Definição 1.4: Uma matriz $M$ é dita pseudo-hermitiana se existe uma outra matriz $\eta$ que satisfaça a seguinte relação (similaridade):

$$
M^{\dagger}=\eta \cdot M \cdot \eta^{-1}
$$

A Teoria de probabilidade e a álgebra linear são as bases matemáticas na qual a mecânica quântica está estruturada, com a teoria das matrizes indispensável para este estudo.

\subsection{Teoria das Matrizes Aleatórias (RMT)}

Podemos combinar teoria de probabilidades com álgebra linear para produzir uma área na matemática denominada Teoria das Matrizes Aleatórias (RMT, do inglês) [5]. Uma matriz aleatória é aquela onde seus elementos de entrada são sorteados a partir de uma função densidade de probabilidades (pdf) para uma variável aleatória. O problema central da RMT é obter o espectro de uma matriz (autovalores) a partir da distribuição dos elementos de entrada. Sob certas condições, essa pode ser uma tarefa difícil, e em alguns casos, impossível de ser performada. Uma classificação muito útil é a que foi proposta por Layman [5] que é baseada na simetria que determinado grupo de matrizes (Ensemble) possuem. Para os casos apresentado na figura a seguir, a tarefa de obter o espectro a partir da distribuição conjunta dos elementos de entrada, pode ser realizada. 


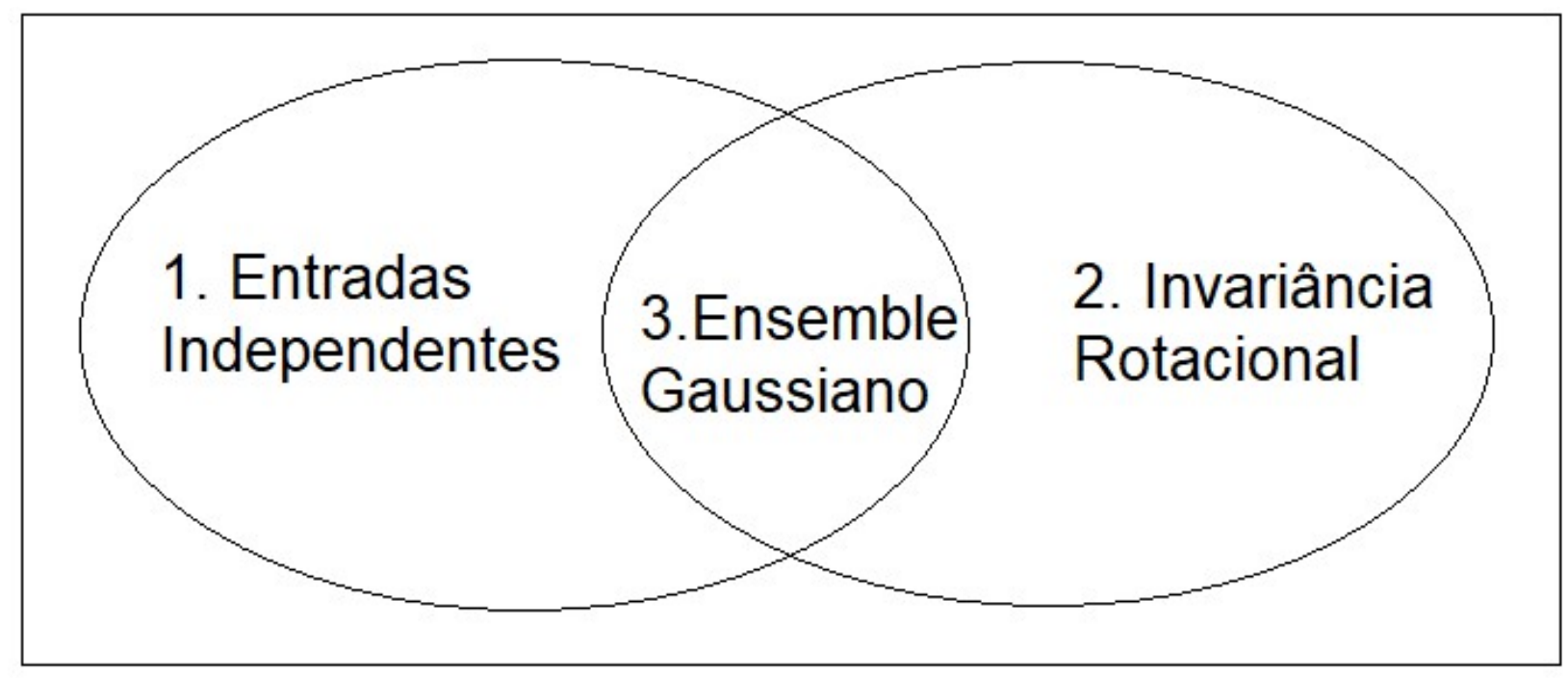

Figura 1: Classificação de Layman de acordo com a simetria do ensemble, figura adaptada de [5].

As matrizes $M$, com elementos de entrada independentes e identicamente distribuídos (i.i.d), possuem a propriedade da distribuição conjunta de probabilidades $\rho[M]$ poder ser fatorada:

$$
\rho[M]=\prod_{i=i}^{n} \prod_{j=1}^{m} \rho\left[M_{i j}\right] .
$$

O segundo grupo da figura 1 contém as matrizes que possuem a propriedade da invariância rotacional. Em essência, essa propriedade implica que duas matrizes quaisquer relacionadas via uma transformação de similaridade $H^{\prime}=U H U^{-1}$ irão ocorrer com a mesma probabilidade no ensemble [5]

$$
\rho[H] d H=\rho\left[H^{\prime}\right] d H^{\prime}
$$

Com a combinação das propriedades anteriores, elementos de entrada independentes e identicamente distribuídos e invariância rotacional, forma-se o que é conhecido na literatura pelo nome de ensemble Gaussiano.

Para que uma determinada matriz aleatória possa ser consistente com um determinado sistema físico é importante atentar-se ao que ficou conhecido como "threefold way" de Dyson [6,13], representado pelo índice $\beta$. O que basicamente este índice indica é a quantidade de variáveis normais 
que aparecem na entrada de uma matriz aleatória. Para $\beta=1,2,4$ temos que as variáveis são: real, complexa ou quarternion, respectivamente. Os trabalhos que exploram o contínuo do índice de Dyson, formam o que ficou conhecido como $\beta$-ensembles. Os potenciais de cada ensemble, assim como as simetrias envolvidas, estão resumidos na tabela abaixo, que foi adaptada de um importante artigo de I. Dumitriu e A. Edelman [13].

\begin{tabular}{|c|c|c|c|c|}
\hline Ensemble & Potencial & $\boldsymbol{\beta}$ & Conjunto & Simetria \\
\hline \multirow{3}{*}{ Hermite } & \multirow{3}{*}{$\mathrm{V}(\lambda)=e^{-\lambda^{2} / 2}$} & 1 & $\mathbb{R}$ & $A \rightarrow \mathrm{Q}^{T} A Q$ \\
\hline & & 2 & $\mathbb{C}$ & $A \rightarrow \mathrm{U}^{H} A U$ \\
\hline & & 4 & $\mathbb{H}$ & $A \rightarrow S^{D} A S$ \\
\hline \multirow{3}{*}{ Laguerre } & \multirow{3}{*}{$\begin{array}{l}\mathrm{V}(\lambda)=\lambda^{a} \cdot e^{-\lambda / 2} \\
a=\frac{\beta}{2} \cdot(m-n+1)-1\end{array}$} & 1 & $\mathbb{R}$ & $A \rightarrow \mathrm{Q}^{T} A Q$ \\
\hline & & 2 & $\mathbb{C}$ & $A \rightarrow \mathrm{U}^{H} A U$ \\
\hline & & 4 & $\mathbb{H}$ & $A \rightarrow S^{D} A S$ \\
\hline
\end{tabular}

Tabela 1: Ensemble clássicos em matrizes aleatórias

Pelo exposto, podemos observar que existe uma conexão natural entre o problema em teoria de matrizes aleatórias e o problema central em mecânica quântica. Na década de 50, Wigner motivado no estudo do espectro de núcleo de átomos pesados [5,6], aplicou técnicas da RMT. As aplicações, na física, não se restringem apenas a teoria quântica, tendo de fato aumentado para as mais diversas áreas, cromodinâmica quântica e física do estado sólido, por exemplo [7,8,9,10,11].

Este trabalho focou no ensemble $\beta$-Laguerre (classicamente conhecido como ensemble de Wishart) que foi introduzido no trabalho de sistemas multivariados publicado em 1928 pelo estatístico Wishart [12]. Este ensemble contém matrizes quadradas que são obtidas a partir de matrizes retangulares. A primeira propriedade interessante deste ensemble para a física é que os autovalores são positivo definidos, ou seja, existe um autovalor que representa o chamado estado fundamental do sistema. As matrizes deste ensemble são utilizadas para tratar sistemas interagentes. É possível explorar a simetria do ensemble para reduzir as matrizes quadradas do ensemble para uma forma tri-diagonal. Ao invés de sortear números a partir de uma distribuição normal para os elementos de entrada, Dumitriu [13] mostrou 
que nessa nova representação para o ensemble utiliza-se a função densidade de probabilidades $\chi$ (chi). Certos vínculos estabelecem os graus de liberdade da distribuição, esses variam de acordo com a posição do elemento de entrada.

Além de estudar o formalismo clássico presente no ensemble de Wishart, este trabalho tem como parte da originalidade a introdução de uma quebra de simetria nas matrizes de maneira a verificar como o espectro se apresenta. A sequência que está organizada este trabalho é a que segue:

Inicialmente foi feito um estudo analítico da distribuição dos elementos de entrada do ensemble clássico de Wishart, que foi utilizado como referência para o que é apresentado na sequência.

> Conhecendo a distribuição dos elementos de entrada é analisado o problema central em RMT, obter a distribuição de probabilidade dos autovalores.

$>$ Tratei em seguida do ensemble $\beta$-Laguerre representado por matrizes tridiagonais ("tight-binding models"). Foi realizado um estudo analítico a fim de verificar que a distribuição dos autovalores é preservada quando comparada com a matriz cheia.

$>\mathrm{Na}$ parte final, é apresentado um estudo numérico feito para os modelos analíticos anteriores. Esse estudo estende-se para sistemas que é introduzido uma quebra de simetria. Essa quebra, será feita de maneira a verificar o espectro de matrizes que satisfazem a equação (5). 


\section{Capítulo 2}

\section{Ensemble de Wishart}

Neste capítulo será feita uma revisão das principais propriedades estatísticas dos elementos de entrada de uma matriz que pertence ao ensemble clássico de Wishart. Os resultados apresentados nesta seção foram importantes para a determinação das propriedades estatísticas dos autovalores.

\subsection{Introdução}

O ensemble de Wishart, ou $\beta$-Laguerre com $\beta$ sendo um índice do "threefold way"de Dyson, possui a invariância rotacional como simetria: $\rho[W]=\rho\left[U \cdot W \cdot U^{-1}\right]$. Essa propriedade implica que os autovetores, obtidos através da diagonalização de $W$, são invariantes por rotação. Como discutido no capítulo anterior, os elementos, de entrada, das matrizes $H$ são independentes, contudo, a função de probabilidade conjunta dos elementos de $W$ é correlacionada, o que não permite a fatoração dela em termos simples como função apenas de cada elemento individualmente.

Definição 2.1: Seja $H$ uma matriz aleatória retangular no espaço de matrizes de dimensão $n \times m$ ( $n>m$ ) cujos elementos de entrada sejam determinados pelo índice de Dyson $\beta=1,2$ e 4 . Esses elementos são independentes e podem ser real, complexo ou quartenion com densidades gaussianas dadas por

$$
\frac{1}{\sqrt{2 \pi}} e^{-x_{i j}^{2} / 2}, \frac{1}{\pi} e^{-\left|z_{i j}\right|^{2}} \text { e } \frac{2}{\pi} e^{-2\left|\omega_{i j}\right|^{2}}
$$


Nos três casos $\beta=1,2$ e 4 , respectivamente. A matriz aleatória quadrada $W$ que pertencente ao ensemble de Wishart é obtida através da relação $W=H \cdot H^{T}(\beta=1)$ ou $W=H \cdot H^{\dagger}(\beta=2,4)[14]$, onde $\dagger$ representa a conjugação transposta.

\subsection{Densidade de probabilidade conjunta dos elementos de entrada}

Devido ao ensemble Wishart real $(\beta=1)$ ser bem documentado em literatura, daqui por diante quando não houver ambiguidade não será discriminado o índice de Dyson, quando isso ocorrer considere o ensemble Wishart complexo $\left(W_{\beta=2}=W_{2}\right)$.

Definição 2.2.1: O ensemble Wishart complexo é definido por duas propriedades [6]:

I) Sendo $H_{i j}^{R}$ e $H_{i j}^{I}$ as partes real e imaginária de $H_{i j}$, respectivamente, temos que a probabilidade $(P(H) d H)$ de um determinado sistema pertencer ao elemento de volume

$$
\begin{array}{r}
d H=\prod_{i \leq j} d H_{i j}^{0} \prod_{i<j} d H_{i j}^{I} \text { é invariante, ou seja, } \\
P(H) d H=P\left(H^{\prime}\right) d H^{\prime} .
\end{array}
$$

Sobre o conjunto de transformações

$$
H^{\prime}=L H U
$$

Onde $L$ e $U$ correspondem respectivamente as matrizes quadradas não singulares sobre o plano complexo de rank $m$ e as matrizes unitárias quadradas de rank $n$.

II) A densidade de probabilidade $P(H)$ pode ser fatorada, devido a independência dos elementos, na forma,

$$
P(H)=\prod_{i \leq j} f_{i j}^{R}\left(H_{i j}^{R}\right) \prod_{i<j} f_{i j}^{I}\left(H_{i j}^{I}\right)
$$

onde as funções $f$ dependem de uma única variável. 
Teorema 2.2.1: Seja $W$ uma matriz no ensemble de Wishart, temos que a distribuição de probabilidade dos elementos de entrada é dada por:

$$
\rho[W]=\frac{1}{Z_{n \beta}} \cdot(\operatorname{det} W)^{\frac{\beta}{2}(m-n)-1} e^{-(1 / 2) \operatorname{Tr}(W)}
$$

A constante de normalização $Z_{n \beta}$ desempenha papel análogo ao da função de partição em mecânica estatística. De fato, muitas propriedades termodinâmicas podem ser exploradas a partir desta constante [6].

Demonstração: Podemos escrever a densidade de probabilidade dos elementos como

$$
\rho[W]=\int d H \rho[H] \delta\left(W-H H^{\dagger}\right)
$$

Na equação acima a medida $d H$ representa que a integração é performada sobre todos os graus de liberdade de $W$, e a delta é apenas um vínculo que força a igualdade entre as duas matrizes. Para realizar a integração acima, vamos inicialmente considerar os seguintes fatos matemáticos.

Definição 2.2.2: Para uma matriz complexa $n \times m \quad \mathrm{Z}=\left[z_{i j}\right] \in \mathbb{C}^{n \times m}$, a respectiva função delta é definida como [15],

$$
\delta(Z):=\prod_{i=1}^{n} \prod_{j=1}^{m} \delta\left(\operatorname{Re}\left(z_{i j}\right)\right) \delta\left(\operatorname{Im}\left(z_{i j}\right)\right)
$$

Proposição 2.2.1: Para uma matriz hermitiana complexa $X \in \mathbb{C}^{n \times n}$ temos,

$$
\delta(X)=\frac{1}{2^{n} \pi^{n^{2}}} \int d T \cdot e^{i T r(T X)}
$$

Onde $T=\left\{t_{i j}\right\}$ também é uma matriz hermitiana complexa de rank $n$ e a medida de integração

$$
d T=\prod_{i=1}^{n} d t_{i i} \prod_{i<j}^{n} d \operatorname{Re}\left(t_{i j}\right) d \operatorname{Im}\left(t_{i j}\right)
$$


é sobre todos os graus de liberdade de $T$.Para uma prova da preposição anterior consultar referência [15].

Vale lembrar que, devido a independência dos valores de entrada da matriz $H$ também podemos escrever:

$$
\rho[H]=\prod_{i=1}^{n} \prod_{j=1}^{m}\left[\frac{1}{2 \pi} e^{-\frac{1}{2}\left\{\left(H_{i j}^{R}\right)^{2}+\left(H_{i j}^{I}\right)^{2}\right\}}\right]
$$

Que pode elegantemente ser reescrito como função do traço.

$$
\rho[H]=\left(\frac{1}{2 \pi}\right)^{n m} e^{-\frac{1}{2} \operatorname{Tr}(H H \dagger)}
$$

Podemos combinar as equações (2.2.7), (2.2.6) e (2.2.3) para escrever,

$$
\rho[W]=\left(\frac{1}{2^{n} \pi^{n^{2}}}\right) \cdot\left(\frac{1}{2 \pi}\right)^{n m} \int d T \int d H \cdot e^{-\frac{1}{2} \operatorname{Tr}\left(H H^{\dagger}\right)+i \operatorname{Tr}\left(T \cdot\left(W-H H^{\dagger}\right)\right)}
$$

Para realizar a primeira integração foi utilizada a identidade [5],

$$
\left[\operatorname{det}\left(\mu 1_{n \times n}-T\right)\right]^{-m}=\frac{1}{(4 \pi i)^{m n}} \int \prod_{k=1}^{m} \prod_{i=1}^{n} d v_{k i} d v_{k i}^{*} e^{\left\{\frac{i}{2} \mu \sum_{k=1}^{m} v_{k}^{\dagger} v_{l}-\frac{i}{2} \sum_{k=1}^{m} v_{k}^{\dagger} T v_{l}\right\}}
$$

Onde $v_{k}$ representa vetores (colunas) complexos e temos que $\operatorname{Im}(\mu)>0$. Pode-se facilmente ver que para realizar a integração em $H$ precisa-se primeiramente realizar uma mudança de variáveis dada por $\left(H_{i j}^{R}, H_{i j}^{I}\right) \rightarrow\left(v_{k i}, v_{k i}^{*}\right)$. Podemos definir as novas variáveis como função das antigas através das relações: 


$$
\left\{\begin{array}{l}
H_{i k}^{R}=\frac{1}{2}\left(v_{k i}+v_{k i}^{*}\right) \\
H_{i k}^{I}=\frac{1}{2 i}\left(v_{k i}^{*}-v_{k i}\right)
\end{array}\right.
$$

O que nos leva ao Jacobiano (para cada grau de liberdade),

$$
J=\operatorname{det}\left(\begin{array}{ll}
\frac{\partial H_{i j}^{R}}{\partial v_{k i}} & \frac{\partial H_{i j}^{R}}{\partial v_{k i}^{*}} \\
\frac{\partial H_{i j}^{I}}{\partial v_{k i}} & \frac{\partial H_{i j}^{I}}{\partial v_{k i}^{*}}
\end{array}\right)=\operatorname{det}\left(\begin{array}{cc}
1 / 2 & 1 / 2 \\
1 / 2 i & -1 / 2 i
\end{array}\right)=\left|-\frac{1}{2 i}\right|
$$

Dividindo cada grau de liberdade de $T$ na equação (2.2.8) por $1 / 2$, podemos reescrever parte da integral (2.2.8) como,

$$
\int d T e^{-\frac{i}{2} T r(T W)} \int d H \cdot e^{-\frac{1}{2}\left(\operatorname{Tr}\left(H H^{\dagger}\right)+i T r\left(-H T H^{\dagger}\right)\right)}=\left(\frac{1}{2}\right)^{n m} \int d T e^{-\frac{i}{2} \operatorname{Tr}(T W)} \int \prod_{k=1}^{m} \prod_{i=1}^{n} d v_{k i} d v_{k i}^{*} e^{\left\{\frac{i^{2}}{2} \sum_{k=1}^{m} v_{k}^{\dagger} v_{l}-\frac{i}{2} \sum_{k=1}^{m} v_{k}^{\dagger} T v_{l}\right\}}
$$

Aplicando a identidade (2.2.9) segue imediatamente que,

$$
\rho[W]=\left(\frac{1}{2^{n} \pi^{n^{2}}}\right) \cdot\left(\frac{1}{2 \pi}\right)^{n m}\left(\frac{1}{2}\right)^{n^{2}+n m}(4 \pi i)^{n m} \int d T e^{-\frac{i}{2} \operatorname{Tr}(T W)}\left[\operatorname{det}\left(i 1_{n \times n}-T\right)\right]^{-m}
$$

A expressão acima é um caso particular da conhecida integral de segundo tipo de Ingham-Siege [16]l, que é dada de maneira geral pela expressão,

$$
J_{n m}(Q, \mu)=\int d T \cdot e^{i T r(T Q)}\left[\operatorname{det}\left(T-\mu 1_{n \times n}\right)\right]^{-m}
$$


Consultando uma tabela de integrais, temos diretamente o resultado apresentado no teorema 2.2.1, o que concluí a prova. 


\section{Capítulo 3}

\section{Estatística dos Autovalores}

Neste capítulo será estudada a estatística dos autovalores do ensemble complexo de Wishart, formado por matrizes simétricas $W_{n n}=H_{n m} \cdot H_{m n}^{\dagger}$ onde $m>n$. Partindo da distribuição conjunta de probabilidades, para os autovalores, será utilizada a técnica dos polinômios especiais para obter a densidade espectral. Finalmente, completando a discussão, é apresentada a forma da distribuição para o limite assintótico $n, m \rightarrow \infty$.

\subsection{Introdução}

Na seção anterior foi apresentada a função densidade de probabilidade para os elementos de uma matriz no ensemble de Wishart. Para o caso complexo desse ensemble, $\beta=2$, utilizando a equação 2.2.11, pode-se escrever:

$$
\rho[W]=\frac{1}{2^{n m} \pi^{\frac{n(n-1)}{2}} \prod_{j=m-n+1}^{m} \Gamma(j)} \cdot(\operatorname{det} W)^{m-n} e^{-(1 / 2) \operatorname{Tr}(W)}
$$

Conforme mencionado anteriormente, o problema central da RMT é obter a densidade de probabilidade do espectro a partir da distribuição dos elementos de entrada. De maneira simplificada, para obtermos a densidade de probabilidade conjunta dos autovalores precisamos aplicar uma transformação $\Xi$ que realiza uma mudança de variáveis, 


$$
\{W\} \rightarrow \Xi(\Lambda, \Upsilon)
$$

onde $\Lambda$ é o termo representando os $n$ autovalores e $\Upsilon$ contém os $\frac{n(n-1)}{2}$ termos adicionais associado aos autovetores, o que totaliza os $\frac{2 n+n(n-1)}{2}$ termos independentes contidos na matriz simétrica $W$. A equação (3.1.2) implica que,

$$
\rho[W] d W=\rho[W(\Lambda, \Upsilon)] \cdot|J(W \rightarrow\{\Lambda, \Upsilon\})| \cdot d \Lambda \cdot d \Upsilon
$$

É bem conhecido em literatura $[5,14,16]$ que o jacobiano da transformação é uma função apenas dos autovalores. Na teoria de matrizes aleatórias, esse determinante é conhecido como determinante de Vandermonde.

$$
|\Delta|^{\beta}:=J(W \rightarrow\{\Lambda, \Upsilon\})=\prod_{i>j}\left(\lambda_{i}-\lambda_{j}\right)^{\beta}
$$

Dessa forma, para se obter a densidade conjunta dos autovalores, basta integrar a parte relativa aos autovetores

$$
\rho[\Lambda]=\int d \Upsilon \cdot \rho[W(\Lambda, \Upsilon)] \cdot|J(W \rightarrow\{\Lambda, \Upsilon\})|
$$

Como os autovetores são invariantes no ensemble de Wishart, ela pode ser absorvida na normalização da função densidade de probabilidade do espectro. Utilizando o procedimento acima, pode se mostrar que a distribuição conjunta de probabilidades dos autovalores é dada por [5,14],

$$
\rho\left(\lambda_{1}, \ldots, \lambda_{n}\right)=\frac{1}{Z_{n, \beta}} \cdot \exp \left[-\frac{1}{2} \sum_{i=1}^{n} \lambda_{i}\right] \prod_{i=1}^{n} \lambda_{i}^{\alpha \beta / 2} \prod_{i \neq j}\left|\lambda_{i}-\lambda_{j}\right|^{\beta}
$$

onde a constante de normalização pode ser obtida empregando-se integrais de Selberg $[13,14,17]$ 


$$
\frac{1}{\mathrm{Z}_{n, \beta}}=2^{-n m \beta / 2} \prod_{i=1}^{n} \frac{\Gamma\left(1+\frac{\beta}{2}\right)}{\Gamma\left(1+\frac{\beta i}{2}\right) \Gamma\left(\frac{\beta m-\beta(n-i)}{2}\right)}
$$

\subsection{Densidade de estados}

Para se obter a densidade espectral, a partir de (3.1.6), precisa-se se calcular o marginal da distribuição.

$$
\rho_{n}\left(\lambda_{1}\right)=\int \ldots \int \prod_{j=2}^{n} d \lambda_{j} \cdot \rho\left(\lambda_{1}, \ldots, \lambda_{n}\right) 3.2 .1
$$

Uma maneira de realizar a múltipla integração acima, consiste em utilizar a técnica dos polinômios especiais. Essa, é bem descrita em literatura de RMT [5,14], e seguindo o procedimento, para o caso de interesse, foi possível encontrar a distribuição espectral para $n$ finito.

Primeiramente, escrevemos a distribuição (3.1.5), para o ensemble complexo de Wishart, como:

$$
\rho\left(\lambda_{1}, \ldots, \lambda_{n}\right)=\frac{1}{Z_{n}} \prod_{i=1}^{n} \exp \left[-2 \cdot V\left(\lambda_{i}\right)\right] \cdot \operatorname{det}\left(\Delta \cdot \Delta^{T}\right) \quad 3.2 .2
$$

onde $V(\lambda)$ denota o potencial de confinamentos dos autovalores, que no ensemble analisado é dado por:

$$
V\left(\lambda_{i}\right)=\frac{\lambda_{i}}{2}-\frac{\alpha \ln \left(\lambda_{i}\right)}{2}
$$

com $\alpha=m-n$. Podemos escrever o determinante de Vandermonde em termos de uma matriz cujas entradas são polinômios a serem fixados $p(x)$. Utilizando a identidade 


$$
\left(\prod_{k} g\left(x_{k}\right)\right) \operatorname{det}\left[f\left(x_{i}, x_{j}\right)\right]=\operatorname{det}\left[\sqrt{g\left(x_{i}\right) \cdot g\left(x_{j}\right)} \cdot f\left(x_{i}, x_{j}\right)\right] \quad 3.2 .4
$$

podemos escrever a distribuição (3.2.2) em termos do Kernel, que é uma função definida da maneira a seguir.

$$
K_{n}(x, y):=\sum_{k=0}^{n-1} \psi_{k}(x) \cdot \psi_{k}(y)
$$

O potencial de confinamento, do ensemble de Wishart, motiva a utilização de polinômios associados de Laguerre, $p(x)=L_{n}^{\alpha}(x)$, de maneira que:

$$
\psi_{k}(x)=e^{-V(x)} L_{k}^{\alpha}(x) \cdot \sqrt{\frac{k !}{(k+\alpha) !}} \cdot 3.2 .6
$$

Explorando a ortogonalidade dos polinômios associados de Laguerre, podemos escrever a propriedade central do Kernel.

$$
\int d y \cdot K_{n}(x, y) \cdot K_{n}(y, z)=K_{n}(x, z) \quad 3.2 .7
$$

A distribuição de probabilidade dos autovalores, em termos do Kernel, fica:

$$
\rho\left(\lambda_{1}, \ldots, \lambda_{n}\right)=\frac{\operatorname{det}\left[K_{n}\left(\lambda_{i}, \lambda_{j}\right)\right]_{1 \leq i, j \leq n}}{Z}
$$

O Kernel é um objeto importante na teoria das matrizes aleatórias, sendo utilizado para obter a correlação entre os autovalores. Podemos utilizar o lema de integração de Dyson-Gaudin para calcular o marginal da distribuição. 
Lema: Considere um vetor $\vec{x}=\left(x_{1}, \ldots, x_{n}\right)$ e uma matriz $M$ cujas entradas são funções $f\left(x_{i}, x_{j}\right)$ de tal maneira que a forma funcional do Kernel é válida para $M$, sob essas condições, para certa medida $d \mu\left(x_{n}\right)$, temos [18]:

$$
\int d \mu\left(x_{n}\right) \operatorname{det}\left[M_{n}\left(\overrightarrow{x_{n}}\right)\right]=\left[\left(\int d \mu(x) f(x, x)\right)-n+1\right] \operatorname{det}\left[M_{n-1}(\vec{y})\right]
$$

a matriz $M_{n-1}$ apresenta a mesma forma funcional de $M_{n}$, sendo a única substituição $\vec{y}=\left(x_{1}, \ldots, x_{n-1}\right)$.

Esse lema é útil para calcular múltiplas integrais, onde de forma geral para um número de iterações $(n-k)$ é válida a relação:

$$
\int \ldots \int d \lambda_{k+1} \ldots d \lambda_{n} \cdot \operatorname{det}\left[K_{n}\left(\lambda_{i}, \lambda_{j}\right)\right]_{1 \leq i, j \leq n}=(n-k) ! \operatorname{det}\left[K_{n}\left(\lambda_{i}, \lambda_{j}\right)\right]_{1 \leq i, j \leq k}
$$

O marginal da distribuição (3.2.8) é obtido com $\mathrm{k}=1, \rho(\lambda)=K_{n}(\lambda, \lambda)$, que é facilmente normalizado fazendo $\mathrm{k}=0$ em 3.2.9. Esses resultados, permitem escrever a densidade espectral, para $n$ finito, como:

$$
\rho_{n}(\lambda)=\frac{1}{n} \sum_{k=0}^{n-1} e^{-\lambda} \cdot x^{\alpha} \cdot\left[L_{k}^{\alpha}(\lambda)\right]^{2} \cdot \frac{k !}{(k+\alpha) !}
$$

\subsection{Limite assintótico}

Na seção anterior, exploramos a densidade espectral para a situação de um $n$ finito. Um caso importante a ser discutido é o limite assintótico $n, m \rightarrow \infty$, nesse, o espectro não apresenta mais mudanças, congela, e os autovalores ocupam as posições dos zeros do polinômio associado de Laguerre [19]. Este limite é tomado de forma a satisfazer o vínculo $\frac{n}{m} \leq 1$. A expressão assintótica é conhecida na 
literatura como distribuição de Marchenko-Pastur. Podemos utilizar expressões para os limites assintóticos dos polinômios ortogonais [15] para avaliar a distribuição 3.2.10 [5,20]. A distribuição de Marchenko-Pastur é bem conhecida na literatura e dada por,

$$
\rho_{M P}(\lambda)=\frac{1}{2 \pi \lambda} \sqrt{\left(\lambda_{+}-\lambda\right)\left(\lambda-\lambda_{-}\right)}
$$

com $\lambda \in\left[\lambda_{+}, \lambda_{-}\right]$. Os extremos da distribuição são dados por $\lambda_{ \pm}=\left(1 \pm c^{-1 / 2}\right)^{2}$, onde $c=\frac{n}{m}[5]$. A distribuição de Marchenko-Pastur pode ser encarada como a distribuição das raízes dos polinômios de Laguerre [19]. 


\section{Capítulo 4}

\section{Matrizes tridiagonais}

Neste capítulo, será apresentada a forma tridiagonal de uma matriz pertencente ao ensemble de Wishart, popularmente conhecido como $\beta$-Laguerre. Será feita uma discussão do caso hermitano e na sequência apresentado o respectivo modelo pseudo-hermitiano.

\subsection{Matrizes tridiagonais, $\beta$-Laguerre, hermitianas.}

Um fato bem conhecido da mecânica quântica é a dificuldade em calcular a função de onda exata para um sistema multi-eletrônico (muitos elétrons). Nessa situação, empregam-se métodos a fim de encontrar uma solução aproximada. Em sistemas fortemente interagentes, como ocorre em física da matéria condensada, utiliza.se matrizes tridiagonais para descrição de casos unidimensionais. Este método permite a construção do modelo que representa a função de onda conjunta dos elementos. Nesses a diagonal e os elementos fora da diagonal descrevem partes diferentes do sistema [21]. A primeira, diagonal, está relacionada com a localização dos elementos e as subdiagonais descrevem as interações de elementos adjacentes. Em teoria de matrizes aleatórias, as matrizes tridiagonais encontraram espaço, inicialmente, nos estudos dos chamados $\beta$-ensembles [13,22]. Esses estudos, consistiram na compreensão da estatística de matrizes $\operatorname{com} \beta$ sendo um parâmetro contínuo, generalização do threefold way de Dyson. Estudos recentes buscam empregar essas matrizes para descrever operadores não hermitianos com espectro real, os chamados operadores pseudo-hermitianos.

Uma matriz quadrada do ensemble de Wishart $\left(W_{n}\right)$ é construída a partir de uma matriz retangular $H_{n m}$, com $n>m$, preenchida com elementos identicamente e independentemente distribuídos (i.i.d.) com entradas Gaussianas, através da relação $W_{n}=H \cdot H^{\dagger}$, em que ${ }^{\dagger}$ denota a transposição conjugada. 
Para realizar a decomposição de valor singular (SVD do inglês) de uma matriz retangular, emprega-se como passo intermediário, a redução desta matriz para a forma bidiagonal [23]. Uma maneira de realizar essa construção é empregando refletores de Householder [23,24,25], o que nos permite enunciar o seguinte teorema.

Teorema 4.1.1 Dada uma matriz retangular $H_{n m}$, com entradas complexas, tal que seja possivel a construção de uma matriz $W_{n}$ no ensemble de Wishart, sua forma bidiagonal é dada por:

$$
B=\left(\begin{array}{cccc}
\chi_{2 a} & & & 0 \\
\chi_{\beta(n-1)} & \chi_{2 a-\beta} & & \\
& \ddots & \ddots & \\
0 & & \chi_{\beta} & \chi_{2 a-\beta(n-1)}
\end{array}\right)
$$

Onde $a=\frac{m \beta}{2}$ e $\chi_{(\bullet)}$ denota elementos sorteados a partir de uma distribuição chi com $(\bullet)$ graus de liberdade.

Para demonstrar o teorema acima será necessário procurar refletores de Houselholder que leve a matriz inicial para a forma desejada. De maneira geral, um refletor deste tipo é descrito por uma matriz $H=\mathrm{I}-2 \vec{v} \cdot \vec{v}^{\dagger}$, onde $\vec{v}$ denota um vetor perpendicular ao plano refletor e I a correspondente matriz identidade.

Demonstração: Podemos escrever a nossa matriz original de forma conveniente como sendo composta de dois elementos com entradas i.i.d.

$$
H_{n m}=\left(\begin{array}{c}
\vec{x}_{1 m} \\
M_{n-1, m}
\end{array}\right)
$$


inicialmente procuramos um refletor a direita que satisfaça a seguinte relação

$$
R^{T} \cdot \vec{x}=\|x\|(1,0, \ldots, 0)=\|x\| \hat{e}_{1},
$$

sendo que esse é independente da matriz $M_{n-1, m}$ podemos concluir que a aplicação deste refletor produz:

$$
H_{n m} R=\left(\begin{array}{lc}
\|\vec{x}\| & 0 \\
\vec{y}_{n-1,1} & M_{n-1, m-1}^{\prime}
\end{array}\right) .
$$

O vetor coluna $\vec{y}$ e a matriz $M^{\prime}$ preenchidos com elementos i.i.d. Buscamos agora um refletor a esquerda que zere os elementos abaixo da primeira entrada do vetor $\vec{y}$. Naturalmente, esse refletor deve preservar o restante da estrutura obtida até o momento. Escrevendo esse refletor como,

$$
\left(\begin{array}{cc}
1 & 0 \\
0 & L_{n-1, n-1}
\end{array}\right)
$$

tal que

$$
L \vec{y}=\|\vec{y}\| \cdot\left(\begin{array}{c}
1 \\
\vdots \\
0
\end{array}\right)
$$

temos como resultado dessa primeira iteração,

$$
\left(\begin{array}{cc}
1 & 0 \\
0 & L_{n-1, n-1}
\end{array}\right) H_{n m} R=\left(\begin{array}{cc}
\|\vec{x}\| & 0 \\
\|\vec{y}\| & M_{n-1, m-1}^{\prime}
\end{array}\right) .
$$


Como os módulos acima são os comprimentos de gaussianas multivariadas com graus de liberdade $2 a$ e $\beta(m-1)$, para $\|\vec{x}\|$ e $\|\vec{y}\|$ as distribuições de probabilidades destes elementos são dadas por $\chi_{2 a}$ e $\chi_{\beta(m-1)}$, respectivamente. Esse é um procedimento bem conhecido em álgebra linear numérica e de fácil implementação em softwares como o MatLab ${ }^{\circledR}$. Se repetirmos o procedimento acima na matriz $M^{\prime}$ e assim sucessivamente $n-1$ vezes, encontramos a matriz apresentada no teorema 4.1.1, o que conclui a prova.

Conhecendo a forma bidiagonal da matriz $H$, podemos imediatamente construir a matriz tridiagonal $T$ que pertence ao ensemble de Wishart através do produto $T=B \cdot B^{T}$. Como mencionado anteriormente, a bidiagonalização permite obter a SVD da matriz tridiagonal, e com isso obter a distribuição de probabilidade conjunta dos autovalores. Esse resultado foi publicado em um importante artigo por Dumitriu e Edelman, onde o resultado é apresentado a seguir como teorema e a prova pode ser obtida em [13].

Teorema 4.1.2: Considerando que a decomposição espectral de T seja dada por $T=Q D Q^{T}$, onde os sinais da primeira linha de $Q$ são fixados de forma a serem positivos e os autovalores são ordenados em ordem crescente na matriz diagonal $\mathrm{D}$. Temos, que a primeira linha de $Q$ e a matriz $D$ são independentes. A distribuição conjunta de probabilidade dos autovalores é dada por,

$$
\rho\left(\lambda_{1}, \ldots, \lambda_{n}\right)=c_{\beta, a} \cdot \exp \left[-\frac{1}{2} \sum_{i=1}^{N} \lambda_{i}\right] \prod_{i=1}^{N} \lambda_{i}^{\frac{\frac{\beta n-\beta(m-1)}{2}-1}{2}} \prod_{i \neq j}\left|\lambda_{i}-\lambda_{j}\right|^{\beta}
$$

a constante de normalização é dada pela expressão,

$$
c_{\beta, a}=\frac{\prod_{i=1}^{m-1} \Gamma\left(\frac{i \beta}{2}\right) \prod_{i=1}^{m} \Gamma\left(a-\frac{\beta}{2}(i-1)\right)}{2^{2 m-1}} .
$$




\subsection{Matrizes tridiagonais pseudo - hermitianas}

Em mecânica quântica, dado um operador hamiltoniano, que descreve um sistema, busca-se a diagonalização dele. Nesse processo, é conveniente encontrar uma base de autofunções para $H$ que seja simultaneamente autofunções de outros operadores, normalmente observáveis. Para operadores hermitianos [1,2], ou seja, àqueles que correspondem ao seu adjunto

$$
H=H^{\dagger}, \quad 4.2 .1 \text {. }
$$

a condição de uma mesma base é verificada se a seguinte relação de comutação for satisfeita $[H, O]=0$

Bender e colaboradores [26], em estudos recentes, estudaram hamiltonianas na forma

$$
H=p^{2}+x^{2}(i x)^{v}, v \geq 0 \quad 4.2 .2
$$

que embora não satisfaçam 4.2.1, não são simétricas, produzem espectro real. A realidade do espectro é garantida por uma simetria presente no sistema, a chamada simetria $P T$. Essa simetria implica que o sistema é invariante por reflexão espacial, paridade (P), e por inversão temporal (T). É importante salientar que a hamiltoniana deve ser invariante pelas duas simetrias e não a cada uma individualmente, ou seja, $[H, P T]=0$. Esse estudo motivou uma extensão da mecânica quântica para incluir sistemas PTsimétricos $[4,27,28]$.

Definição: Um operador é chamado pseudo-hermitiano, se ele está conectado com seu adjunto via transformação de similaridade, ou seja,

$$
H=\eta \cdot H^{\dagger} \cdot \eta^{-1} \quad 4.2 .3
$$

o que implica que operador e seu adjunto compartilham o mesmo conjunto de autovalores [21]. 
A condição de pseudo-hermiticidade implica que o espectro da matriz precisa ser real ou complexo conjugado, $H \eta|\psi\rangle=h|\psi\rangle=\eta H^{\dagger}|\psi\rangle=h^{*}|\psi\rangle, h \in \mathbb{C}$.

As matrizes tridiagonais, além de possibilitar a generalização do threefold way de Dyson [13], são uma importante ferramenta para o estudo de sistemas pseudo-hermitianos. O ensemble de Wishart apresenta-se como ferramenta eficiente na descrição de sistemas fortemente interagentes. Devido as propriedades desse ensemble, que são interessantes do ponto de vista de sistemas físicos, motivou-se uma extensão análoga àquela feita para sistemas triadiagonais pseudo-hermitianos do ensemble gaussiano [6].

Sabemos, de resultados anteriores, como proceder para obter uma matriz tridiagonal que pertença ao ensemble de Wishart. Para estudar o correspondente caso pseudo-hermitiano será construída uma matriz $T^{\prime}$, da seguinte forma [29,30]:

$$
T^{\prime}=B \cdot C^{T}=\left(\begin{array}{cccc}
a_{1} & & & 0 \\
d_{1} & a_{2} & & \\
& \ddots & \ddots & \\
0 & & d_{n-1} & a_{n}
\end{array}\right) \cdot\left(\begin{array}{cccc}
a_{1} & & & 0 \\
e_{1} & a_{2} & & \\
& \ddots & \ddots & \\
0 & & e_{n-1} & a_{n}
\end{array}\right)^{T}, \quad 4.2 .4
$$

com os elementos da diagonal sorteados a partir de uma distribuição $\chi_{2 a-\beta(m-1)}$ e os da sub-diagonal inferior de uma distribuição $\chi_{\beta(m-1)}$, é importante notar que $d_{i}$ e $e_{i}$ são sorteados independentemente. A matriz construída na forma 4.2.4 é tridiagonal,

$$
T^{\prime}=\left(\begin{array}{cccccc}
a_{1}^{\prime} & b_{1} & 0 & \cdots & 0 & 0 \\
c_{1} & a_{2}^{\prime} & b_{2} & \cdots & 0 & 0 \\
0 & c_{2} & a_{3}^{\prime} & \cdots & 0 & 0 \\
\vdots & \vdots & \vdots & \ddots & \vdots & \vdots \\
0 & 0 & 0 & \cdots & a_{n-1}^{\prime} & b_{n-1} \\
0 & 0 & 0 & \cdots & c_{n-1} & a_{n}^{\prime}
\end{array}\right)
$$


e satisfaz o teorema a seguir.

Teorema 4.2.5: A matriz T'é pseudo-hermitiana.

Demonstração: Precisamos mostrar que T' satisfaz a equação 4.2.3, para isso podemos definir a matriz diagonal $(\eta)$, como sendo:

$$
\eta=\left(\begin{array}{cccc}
1 & 0 & \cdots & 0 \\
0 & \frac{b_{n-1}}{c_{n-1}} & 0 & 0 \\
\vdots & \vdots & \ddots & 0 \\
0 & 0 & 0 & \frac{b_{n-1} \cdot b_{n-1} \cdot b_{1}}{c_{n-1} \cdot c_{n-1} \cdot c_{1}}
\end{array}\right)
$$

o que rapidamente permite escrevermos a matriz inversa como,

$$
\eta^{-1}=\operatorname{diag}\left(1, \frac{c_{n-1}}{b_{n-1}}, \cdots, \frac{c_{n-1} \cdot c_{n-1} \cdot c_{1}}{b_{n-1} \cdot b_{n-1} \cdot b_{1}}\right)
$$

Sendo a matriz $M=\left(T^{\prime}\right)^{T} \cdot \eta^{-1}$, podemos reconhecer seus elementos através da relação,

$$
M_{i j}=\sum_{k=1}^{n} T_{k i}\left(\eta^{-1}\right)_{k j} \delta_{k j}=T_{j i}^{\prime}\left(\eta^{-1}\right)_{j}
$$

onde a delta de Kronecker, definida como 


$$
\delta_{i j}=\left\{\begin{array}{l}
1, i=j \\
0, \mathrm{i} \neq \mathrm{j}
\end{array}\right.
$$

garante a forma diagonal da matriz $\eta^{-1}$. Finalmente, completando o produto matricial, e lembrando da forma tridiagonal de $T^{\prime}$, encontramos a relação,

$$
(\eta M)_{i j}=\eta_{i} T_{j i}^{\prime} \eta_{j}^{-1}=T_{i j}^{\prime}
$$

o que concluí a prova.

Podemos mostrar que a matriz $T^{\prime}$ 'satisfaz um teorema adicional.

Teorema 4.2.6: Se os produtos $b_{i} c_{i}$ são positivos, então o espectro de $T^{\prime}$ é real.

Demonstração: Suponha que T' seja uma matriz não hermitiana que satisfaça 4.2.3 e considere uma matriz $\mathrm{K}$ tal que,

$$
K=\eta^{-1 / 2} \cdot T^{\prime} \cdot \eta^{1 / 2}
$$

isso permite escrever:

$$
K=\eta^{-1 / 2} \cdot T^{\prime} \cdot \eta^{1 / 2}=\eta^{-1 / 2} \cdot\left(\eta \cdot T^{\prime \dagger} \cdot \eta^{-1}\right) \cdot \eta^{1 / 2}
$$

utilizando 4.2.3, temos que

$$
K=\eta^{1 / 2} \cdot T^{\dagger} \cdot \eta^{-1 / 2}=K^{\dagger},
$$


o que implica que $K$ e $T^{\prime}$ compartilham o mesmo conjunto de autovalores, que decorrente da hermiticidade, são reais. Se definirmos a matriz $\eta^{1 / 2}$ como:

$$
\eta^{1 / 2}=\left(\begin{array}{cccc}
\sqrt{1} & 0 & \cdots & 0 \\
0 & \sqrt{\frac{b_{n-1}}{c_{n-1}}} & 0 & 0 \\
\vdots & \vdots & \ddots & 0 \\
0 & 0 & 0 & \sqrt{\frac{b_{n-1} \cdot b_{n-1} \cdot b_{1}}{c_{n-1} \cdot c_{n-1} \cdot c_{1}}}
\end{array}\right)
$$

segue imediatamente que a inversa tem a forma,

$$
\eta^{-1 / 2}=\left(\begin{array}{cccc}
\sqrt{1} & 0 & \cdots & 0 \\
0 & \sqrt{\frac{c_{n-1}}{b_{n-1}}} & 0 & 0 \\
\vdots & \vdots & \ddots & 0 \\
0 & 0 & 0 & \sqrt{\frac{c_{n-1} \cdot c_{n-1} \cdot c_{1}}{b_{n-1} \cdot b_{n-1} \cdot b_{1}}}
\end{array}\right)
$$

o que garante o resultado desejado e concluí a prova.

No capítulo seguinte será feito um estudo numérico de matrizes $T^{\prime}$ a fim de comparar o espectro desta com uma matriz hermitiana tridiagonal que pertença ao ensemble de Wishart. 


\section{Capítulo 5}

\section{Resultados Numéricos}

Neste capítulo, serão apresentados os resultados numéricos encontrados para as principais propriedades estatísticas discutidas nos capítulos precedentes. Iniciamos com uma revisão do ensemble de Wishart clássico, composto por matrizes retangulares cheias, para em seguida analisar os casos tridiagonais hermitianos e pseudo-hermitianos, respectivamente. A fim de complementar a discussão, serão apresentados alguns resultados para o ensemble Anti-Wishart pseudo-hermitiano assim como uma motivação para a extensão deste trabalho introduzindo quebra de hermiticidade e pseudo-hermiticidade.

\subsection{Introdução}

A fim de facilitar a visualização dos principais resultados apresentados na forma de teorema nos capítulos anteriores, foi feita uma investigação numérica para o ensemble de Wishart com $\beta=2$. A vantagem da utilização de cálculo numérico é a possibilidade de estudar limites assintóticos do ensemble, assim como a estatística de elementos centrais. O estudo foi realizado no software MatLab $^{2 \circledR}$ da MathWorks, que possui várias bibliotecas úteis para o estudo realizado. Com o auxílio das bibliotecas disponíveis, foi possível o desenvolvimento de um código numérico que permitisse obter de maneira sistemática os autovalores do sistema desejado. Estes códigos, de autoria própria, foram a base para a criação das figuras apresentadas neste capítulo.

Iniciamos com a comparação do espectro de uma matriz retangular cheia no ensemble de Wishart, com uma tridiagonal. O resultado é importante pois além de confirmar que a forma apresentada para a matriz tridiagonal está correta, permite avaliar o código de programação implementado. No que segue, serão consideradas matrizes tridiagonais hermitianas para comparação com o modelo pseudo-hermitiano.

\footnotetext{
${ }^{2}$ MATLAB, 2018. 8.5.0.197613 (R2015a), Natick, Massachusetts: The MathWorks Inc.
} 
Investigou-se o comportamento estatístico para diferentes valores da constante $c$, definida como $c=\frac{n}{m} \leq 1$.

Como motivação da extensão deste trabalho será realizada a quebra de hermiticidade e pseudohermiticidade via troca de sinal em uma das subdiagonais. Essa troca de sinal na subdiagonal inferior será alternante entre os sinais de $-\mathrm{e}+$, onde o primeiro elemento, $n=2 \mathrm{e} m=1$, é negativo.

\subsection{Desdobramento dos Autovalores}

Para avaliar o espaçamento entre os autovalores foi realizado um procedimento conhecido em literatura de RMT como unfolding, desdobramento dos autovalores. Esse consiste em uma transformação que leva a densidade espectral para uma nova variável que tenha distribuição igual a um [5,31].

$$
\rho_{n}(x) d x=f(\xi) d \xi
$$

tal que

$$
f(\xi)=1
$$

Para expressar a nova variável em termos da antiga podemos realizar a seguinte integração,

$$
\xi(x)=\int^{x} \rho_{n}\left(x^{\prime}\right) d x^{\prime} .
$$

A integral em (5.2.3) não possui uma expressão analítica para todo valor de $n$. Podemos então avaliar essa integral no limite em que $n \rightarrow \infty$, o que implica que a distribuição $\rho_{n}$ tende a distribuição de Marchenko-Pastur. Nesse limite, temos que a nova variável é dada pela expressão [20], 


$$
\xi(x)=\frac{1}{4 \pi}\left[\begin{array}{l}
-4 \sqrt{x_{-} x_{+}} \arctan \sqrt{\frac{x_{+}\left(x-x_{-}\right)}{x_{-}\left(x_{+}-x\right)}}+\left(x_{+}+x_{-}\right) \arccos \left(\frac{x_{+}+x_{-}-2 x}{x_{+}-x_{-}}\right) \\
+2 \sqrt{\left(x_{+}-x\right)\left(x-x_{-}\right)}
\end{array}\right],
$$

com $x_{ \pm}=n(c \pm 1)^{2}$.

Com a transformação acima é possível obter os espaçamentos entre os autovalores, nessa nova variável, das matrizes geradas sem ambiguidade. Isso porque $\xi^{\prime}(x) \geq 0$, obtido de 5.2.1, no intervalo em que estão distribuídos os autovalores, com a igualdade valendo apenas nos extremos, ou seja, em $x_{ \pm}$.

A expressão do perfil do histograma dos espaçamentos dos autovalores é bem conhecida em literatura de RMT: Para um índice de Dyson $\beta$, podemos escrever, [5]

$$
\rho_{\beta}(s)=A \cdot s^{\beta} \cdot e^{-B s^{e}},
$$

onde as constantes $A$ e $B$ são dadas pelas condições de normalização:

$$
\int_{0}^{\infty} \rho_{\beta}(s) \cdot d s=1,
$$

e

$$
\langle s\rangle=\int_{0}^{\infty} \rho_{\beta}(s) \cdot s \cdot d s=1 .
$$

Pode-se facilmente mostrar a partir das equações 5.2.5, 5.2.6 e 5.2.7, que o perfil teórico do histograma de espaçamento de autovalores para o ensemble de Wishart com $\beta=2$ é dado por: 


$$
\rho_{2}(s)=\frac{32}{\pi^{2}} \cdot s^{2} \cdot e^{-\frac{4}{\pi} s^{2}}
$$

\subsection{Ensemble Anti-Wishart}

Estendemos a discussão para o caso do ensemble anti-Wishart, hermitiano e pseudo hermitiano, que consiste nas matrizes $\tilde{W}_{n n}$ obtidas de matrizes retangulares $\tilde{H}_{n m}$ via $\tilde{W}=\tilde{H} \cdot \tilde{H}^{T}$, com $\mathrm{n}>\mathrm{m}$. É possível mostrar que essas matrizes possuem exatamente $(n-m)$ autovalores iguais a zero e que os demais possuem distribuição dada por [32],

$$
\rho^{\text {anti-Wishart }}\left(\lambda_{1}, \ldots, \lambda_{n}\right) \propto \prod_{i} \lambda_{i}^{n-m} e^{-\lambda_{i}} \prod_{i<j}\left(\lambda_{i}-\lambda_{j}\right)^{2}
$$

associados a matriz obtida através da relação $H^{T} H$. Nesse ensemble nem todos os elementos da matriz são aleatórios, possuindo conexão com a primeira linha de $H^{T} H$.

\subsection{Figuras obtidas dos resultados numéricos}

Os resultados numéricos realizados serão aqui sumarizados pelas figuras que seguem. A tabela 5.1 apresenta a identificação correspondente a cada figura, assim como as propriedades de cada uma.

É possível observar, diretamente pelas figuras, as propriedades estatísticas revisadas ao longo do texto, que estão em concordância nos regimes comparados, hermitiano e pseudo-hermitiano. O ensemble de Wishart apresenta-se robusto, no sentido que para diferentes valores de $\beta$, mas com a mesma constante $c$, o perfil apresentado para distribuição de autovalores coincide. Para valores arbitrários de $N$, longe do limite assintótico, a expressão 3.2.10 foi verificada, sendo o caso pseudo-hermitiano mais suscetível a precisão numérica empregada. Observamos diferenças no espaçamento de autovalores para os resultados 
nos casos hermitianos e pseudo-hermitianos. Nesse último, o espaçamento é sempre ajustado por $\beta^{\prime}=2 \cdot \beta$ em 5.2.5, incluindo matrizes fora do threefold way de Dyson. Em um estudo recente da estatística de sistemas pseudo-hermitianos no ensemble de Hermite, M. Pato e O. Bohigas [29], observaram que a função densidade de probabilidades que descreve o sistema pseudo-hermitiano, no limite assintótico de grandes argumentos, tende para a função densidade de probabilidade do caso hermitiano, com a correção em $\beta$ mencionada anteriormente. Esse efeito foi verificado numericamente no estudo realizado para o ensemble de Wishart. Quando impomos a quebra de hermiticidade, e pseudohermiticidade, observamos a mesma distribuição de autovalores no plano complexo. Uma compreensão analítica do perfil observado, para os autovalores, é um resultado a ser investigado como extensão deste trabalho.

Tabela 5.1 - Conteúdo das figuras obtidas via cálculo numérico através do software Matlab ${ }^{\circledR}$.

\begin{tabular}{|c|l|}
\hline Figura & \multicolumn{1}{c|}{ Conteúdo } \\
\hline 5.1 & $\begin{array}{l}\text { Comparação da estatística da frequência de ocorrência de autovalores para matrizes } \\
\text { cheias e tridiagonais hermitianas }(\mathrm{n}=400 \mathrm{~m}=700 \beta=2) .\end{array}$ \\
\hline 5.2 & $\begin{array}{l}\text { Comparação da estatística da frequência de ocorrência de autovalores para matrizes } \\
\text { cheias e tridiagonais hermitianas, com o ajuste da curva da distribuição de } \\
\text { Marchenko-Pastur ( } \mathrm{n}=570 \mathrm{~m}=980 \beta=2) .\end{array}$ \\
\hline 5.3 & $\begin{array}{l}\text { Comparação da estatística da frequência de ocorrência de autovalores para matrizes } \\
\text { cheias e triadiagonais hermitianas, com o ajuste da curva teórica para a distribuição } \\
(\mathrm{n}=5 \text { m=20 } \beta=2) .\end{array}$ \\
\hline 5.4 & $\begin{array}{l}\text { Comparação da estatística da frequência de ocorrência de autovalores para matrizes } \\
\text { tridiagonais hermitianas }(\mathrm{n}=400 \mathrm{~m}=400,500 \beta=2) .\end{array}$ \\
\hline 5.5 & $\begin{array}{l}\text { Comparação da estatística da frequência de ocorrência de autovalores para matrizes } \\
\text { tridiagonais hermitianas ( } \mathrm{n}=400 \mathrm{~m}=700,900,1200 \beta=2) .\end{array}$ \\
\hline 5.6 & $\begin{array}{l}\text { Comparação da estatística da frequência de ocorrência de autovalores para matrizes } \\
\text { tridiagonais pseudo-hermitianas (n=400 } \mathrm{m}=400,500 \beta=2) .\end{array}$ \\
\hline
\end{tabular}




\begin{tabular}{|c|c|}
\hline 5.7 & $\begin{array}{l}\text { Comparação da estatística da frequência de ocorrência de autovalores para matrizes } \\
\text { tridiagonais pseudo-hermitianas }(\mathrm{n}=400 \mathrm{~m}=700,900,1200 \beta=2) \text {. }\end{array}$ \\
\hline 5.8 & $\begin{array}{l}\text { Comparação da estatística da frequência de ocorrência de autovalores para matrizes } \\
\text { tridiagonais hermitianas e pseudo-hermitianas }(\mathrm{n}=5 \mathrm{~m}=20 \beta=2) \text {. }\end{array}$ \\
\hline 5.9 & $\begin{array}{l}\text { Comparação da estatística da frequência de ocorrência de autovalores para matrizes } \\
\text { tridiagonais hermitianas e pseudo-hermitianas }(\mathrm{n}=400 \mathrm{~m}=500 \beta=2) \text {. }\end{array}$ \\
\hline 5.10 & $\begin{array}{l}\text { Comparação da estatística da frequência de ocorrência de autovalores para matrizes } \\
\text { tridiagonais hermitianas e pseudo-hermitianas }(\mathrm{n}=400 \mathrm{~m}=700 \beta=2) \text {. }\end{array}$ \\
\hline 5.11 & $\begin{array}{l}\text { Comparação da estatística da frequência de ocorrência de autovalores para matrizes } \\
\text { tridiagonais hermitianas e pseudo-hermitianas }(\mathrm{n}=400 \mathrm{~m}=500 \beta=0.5) \text {. }\end{array}$ \\
\hline 5.12 & Unfolding de matrizes tridiagonais hermitianas $(\mathrm{n}=400 \mathrm{~m}=700 \beta=2)$. \\
\hline 5.13 & $\begin{array}{l}\text { Comparação da estatística da frequência de ocorrência do espaçamento de } \\
\text { autovalores para matrizes tridiagonais hermitianas e pseudo-hermitianas ( } \mathrm{n}=400 \\
\mathrm{~m}=500 \beta=0.5 \text { ). }\end{array}$ \\
\hline 5.14 & $\begin{array}{l}\text { Comparação da estatística da frequência de ocorrência do espaçamento de } \\
\text { autovalores para matrizes tridiagonais hermitianas e pseudo-hermitianas ( } \mathrm{n}=400 \\
\mathrm{~m}=500 \beta=1) \text {. }\end{array}$ \\
\hline 5.15 & $\begin{array}{l}\text { Comparação da estatística da frequência de ocorrência do espaçamento de } \\
\text { autovalores para matrizes tridiagonais hermitianas }(\mathrm{n}=400 \mathrm{~m}=500 \beta=4) \text { e pseudo- } \\
\text { hermitianas }(\mathrm{n}=400 \mathrm{~m}=500 \beta=2) \text {. }\end{array}$ \\
\hline 5.16 & $\begin{array}{l}\text { Comparação da estatística da frequência de ocorrência do espaçamento de } \\
\text { autovalores para matrizes tridiagonais hermitianas e pseudo-hermitianas ( } \mathrm{n}=400 \\
\mathrm{~m}=700 \beta=2) \text {. }\end{array}$ \\
\hline 5.17 & $\begin{array}{l}\text { Estatística da frequência de ocorrência de autovalores para matrizes tridiagonais } \\
\text { hermitianas e pseudo-hermitianas no ensemble anti-Wishart } \tilde{W}=H \cdot H^{T} \text { (n=500 } \\
\mathrm{m}=200 \beta=2) \text {. }\end{array}$ \\
\hline
\end{tabular}




\begin{tabular}{|c|l|}
\hline 5.18 & $\begin{array}{l}\text { Estatística da frequência de ocorrência de autovalores para matrizes tridiagonais } \\
\text { hermitianas e pseudo hermitanas no ensemble anti-Wishart } \tilde{W}=H^{T} \cdot H \\
(\mathrm{n}=500 \mathrm{~m}=200 \beta=2) .\end{array}$ \\
\hline 5.19 & $\begin{array}{l}\text { Estatística da frequência de ocorrência de autovalores para quebra de hermiticidade } \\
\text { em matrizes tridiagonais hermitianas }(\mathrm{n}=400 \mathrm{~m}=500 \beta=2) .\end{array}$ \\
\hline 5.20 & $\begin{array}{l}\text { Estatística da frequência de ocorrência de autovalores para quebra de pseudo- } \\
\text { hermiticidade (n=400 } \mathrm{m}=500 \beta=2) .\end{array}$ \\
\hline
\end{tabular}




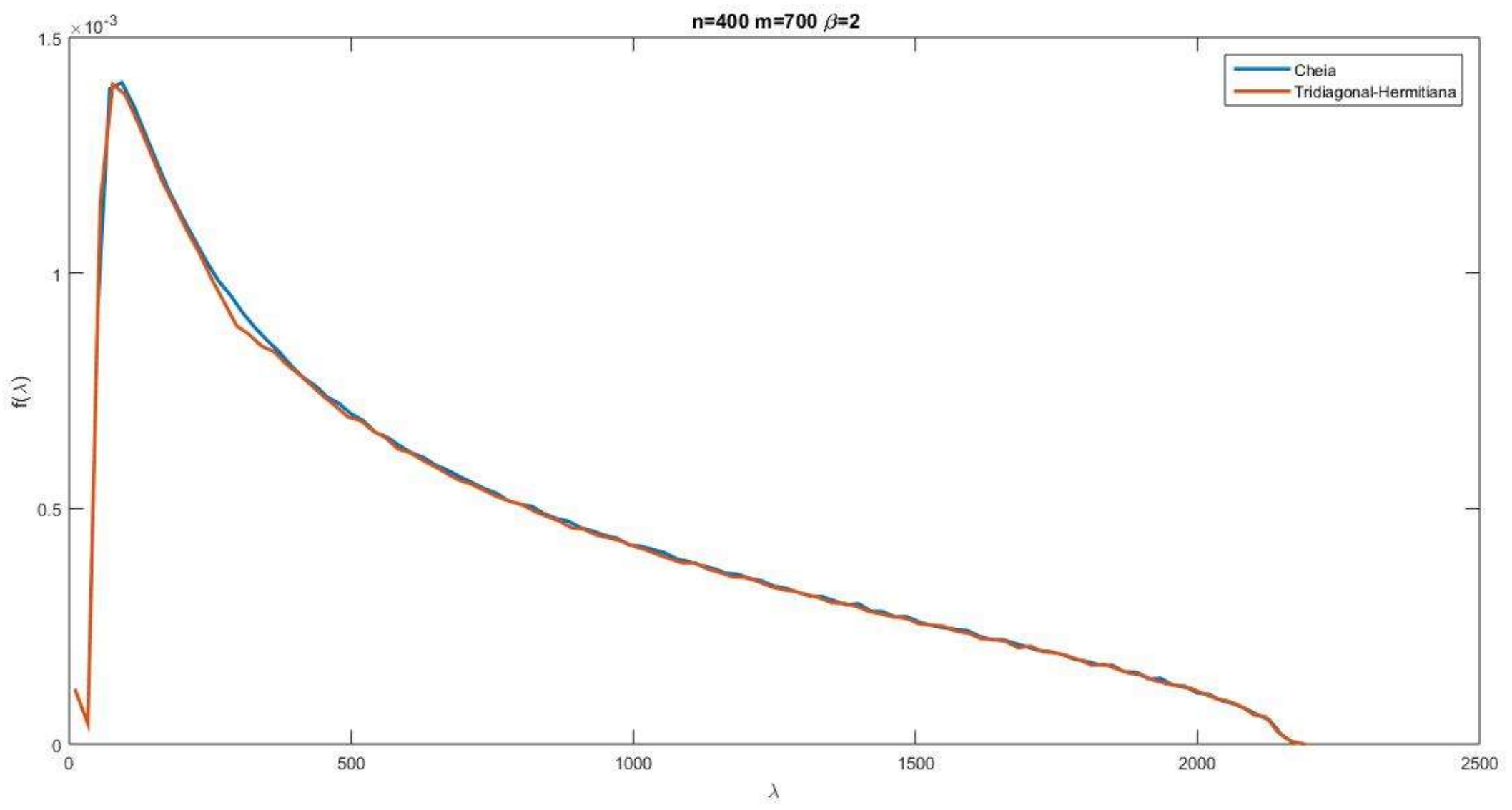

Figura 5.1: Comparação da estatística da frequência de ocorrência de autovalores para matrizes cheias e tridiagonais hermitianas $(\mathrm{n}=400 \mathrm{~m}=700 \beta=2)$. 


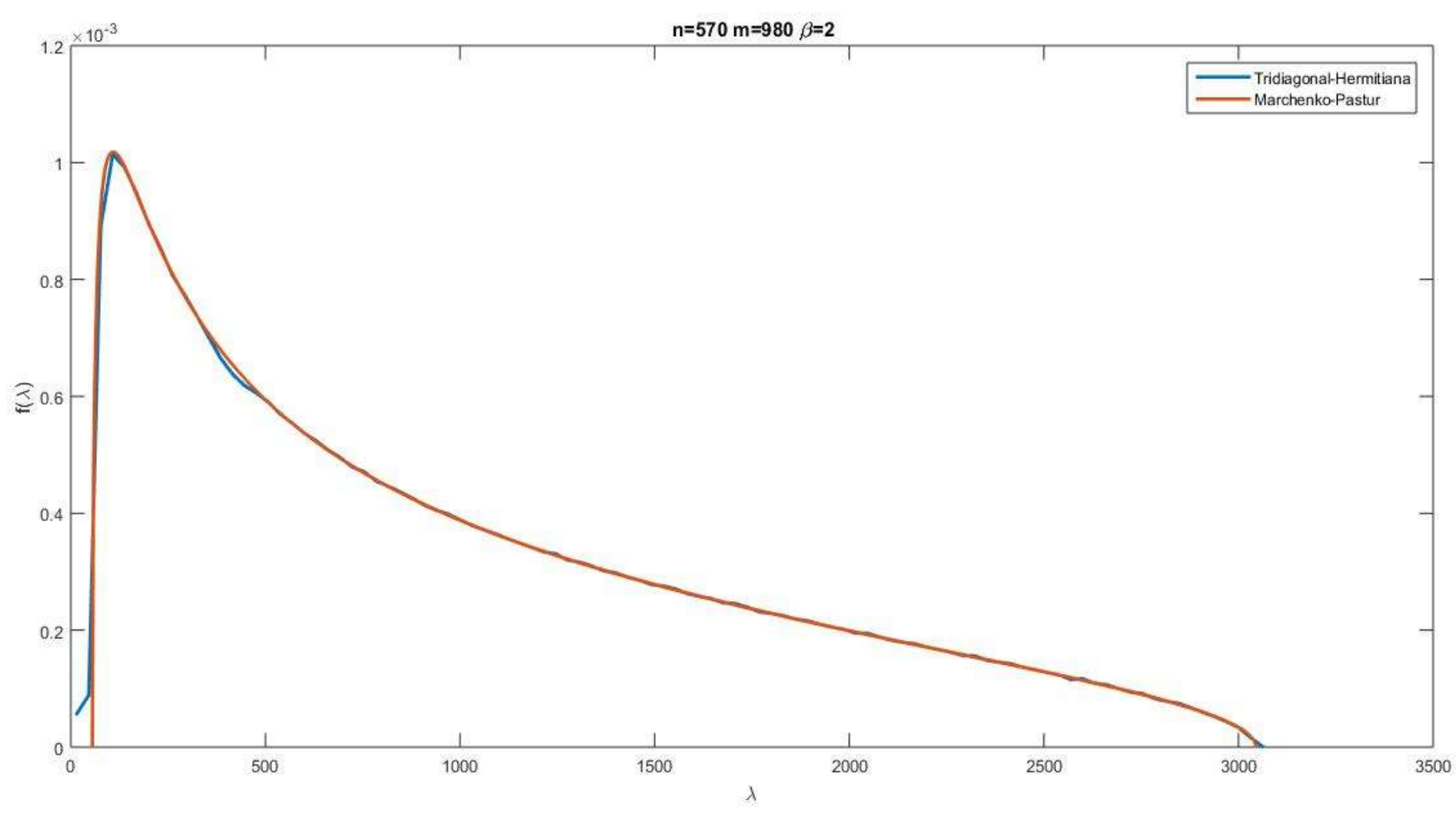

Figura 5.2: Comparação da estatística da frequência de ocorrência de autovalores para matrizes cheias e tridiagonais hermitianas, com o ajuste da curva da distribuição de Marchenko-Pastur ( $\mathrm{n}=570 \mathrm{~m}=980 \beta=2)$. 


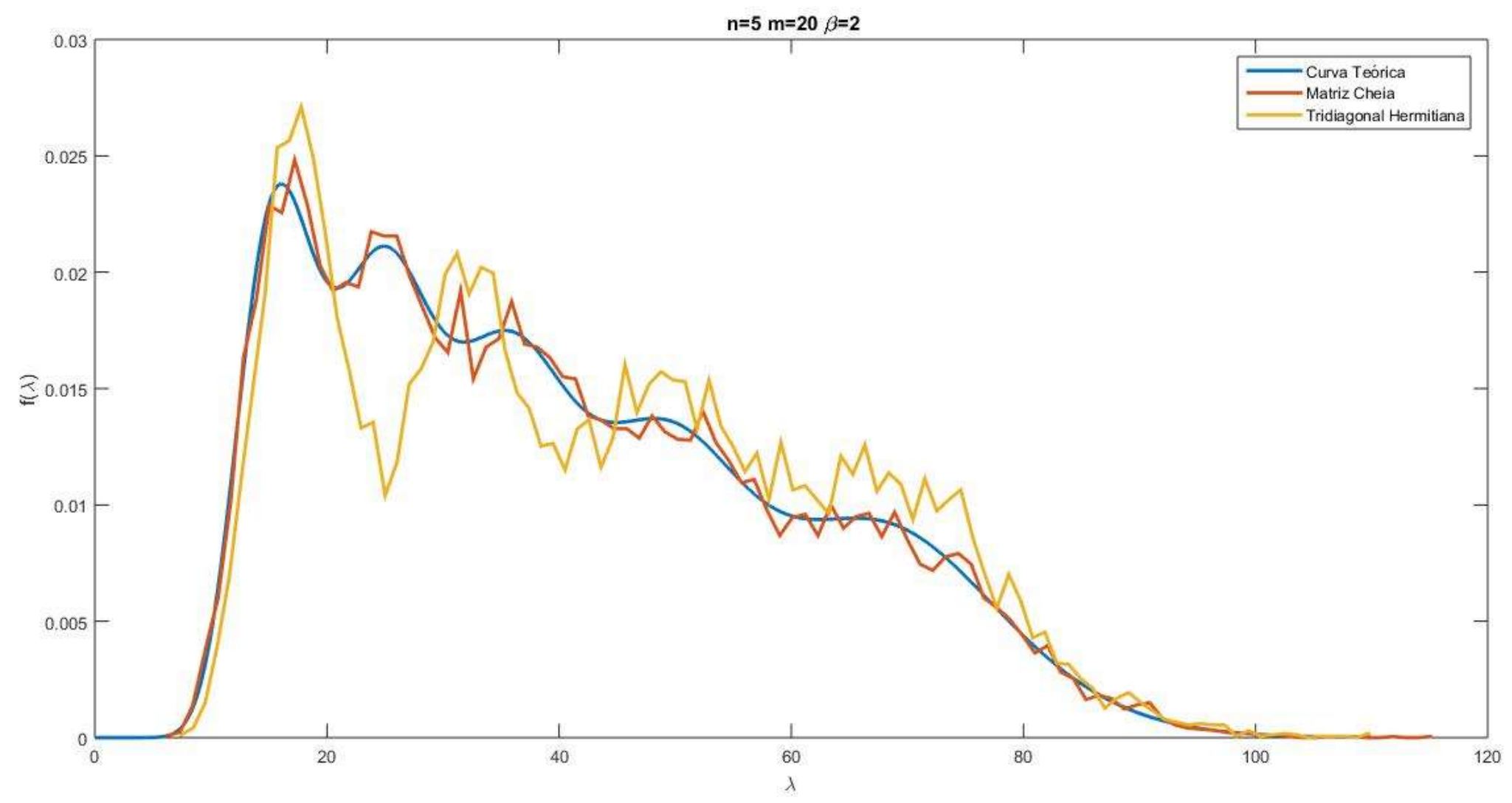

Figura 5.3: Comparação da estatística da frequência de ocorrência de autovalores para matrizes cheias e triadiagonais hermitianas, com o ajuste da curva teórica para a distribuição $(n=5 \mathrm{~m}=20 \beta=2)$. 


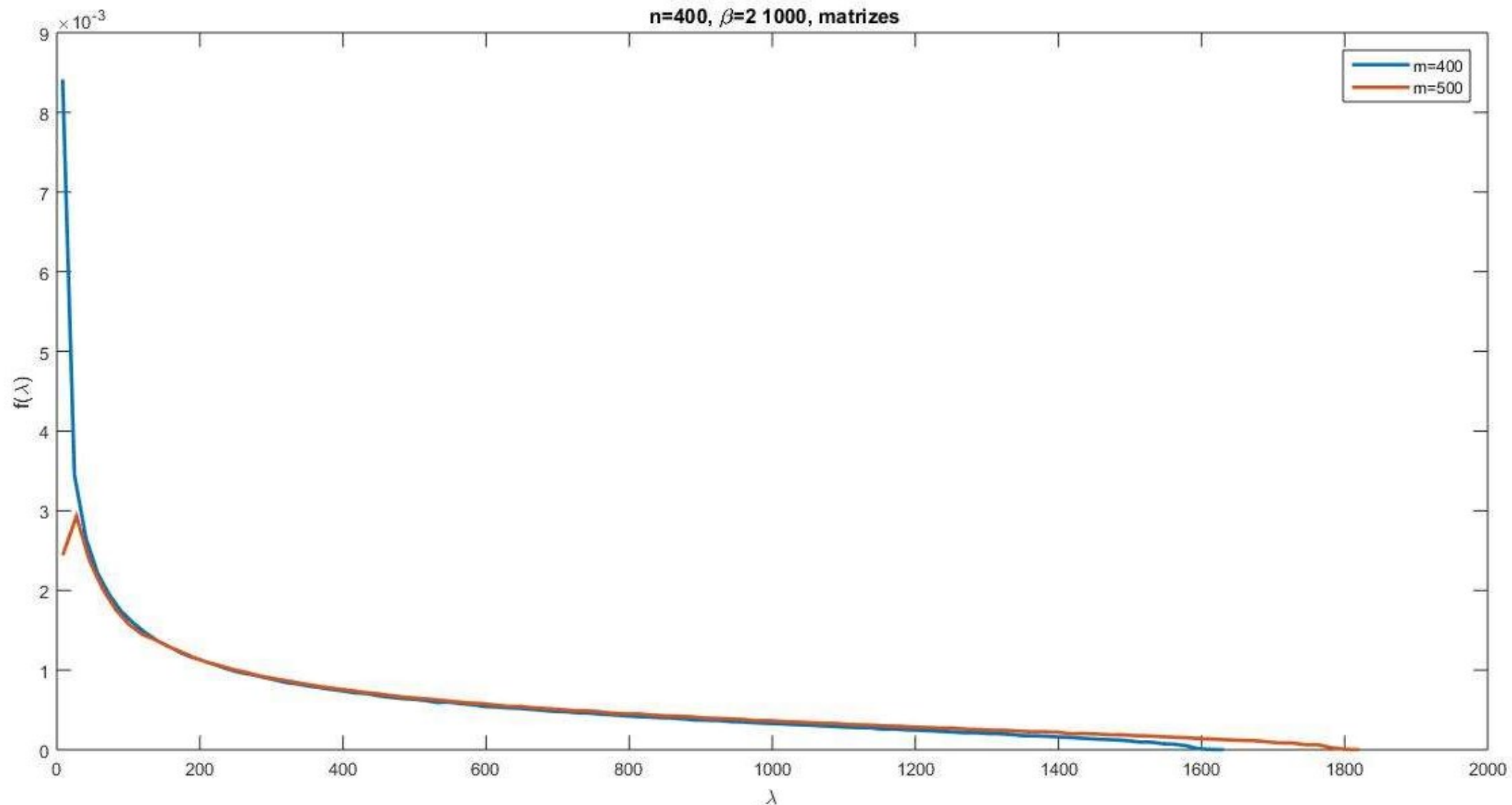

Figura 5.4: Comparação da estatística da frequência de ocorrência de autovalores para matrizes tridiagonais hermitianas

$$
(\mathrm{n}=400 \mathrm{~m}=400,500 \beta=2) .
$$




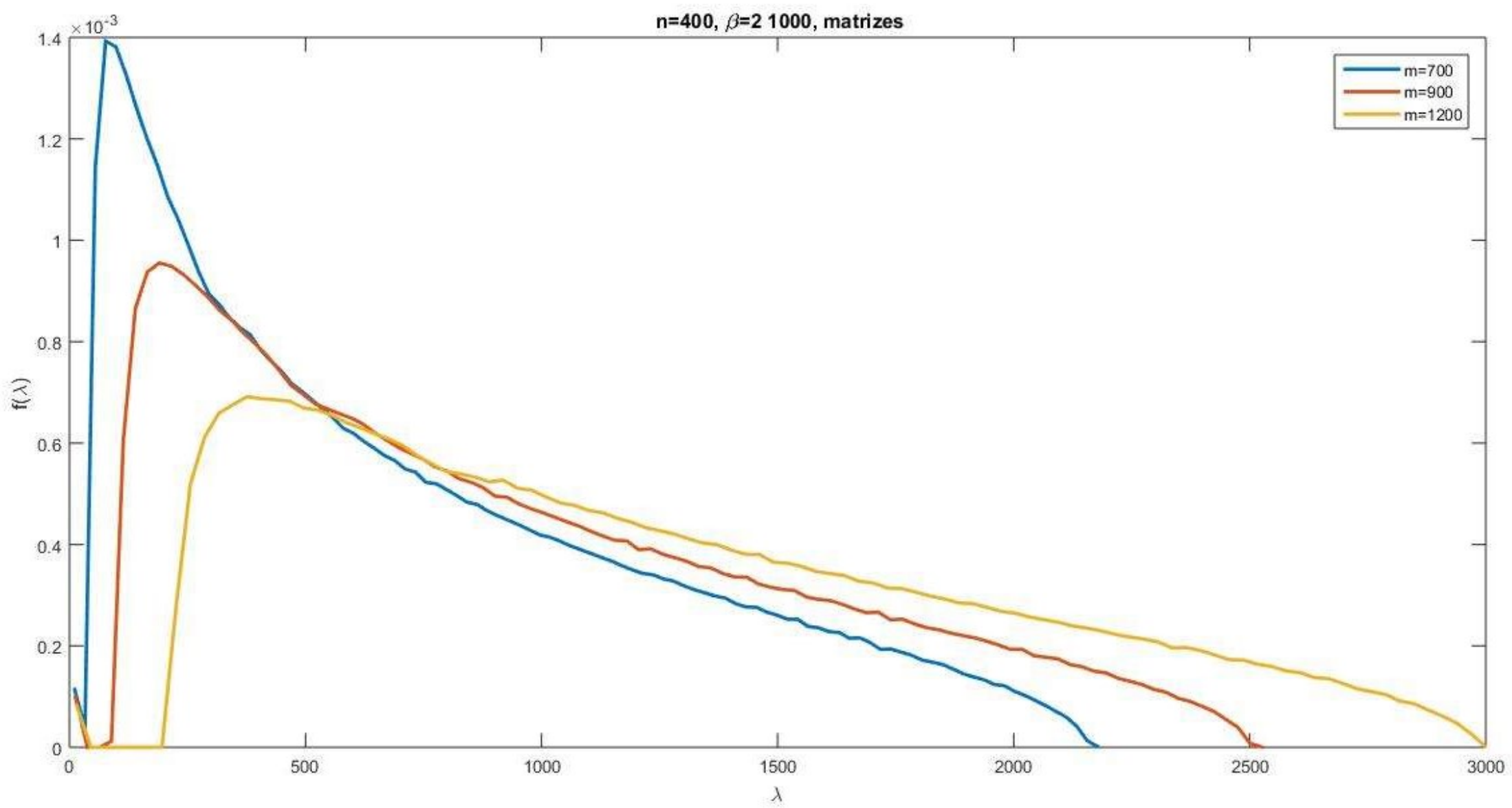

Figura 5.5: Comparação da estatística da frequência de ocorrência de autovalores para matrizes tridiagonais hermitianas ( $\mathrm{n}=400$ $\mathrm{m}=700,900,1200 \beta=2)$. 


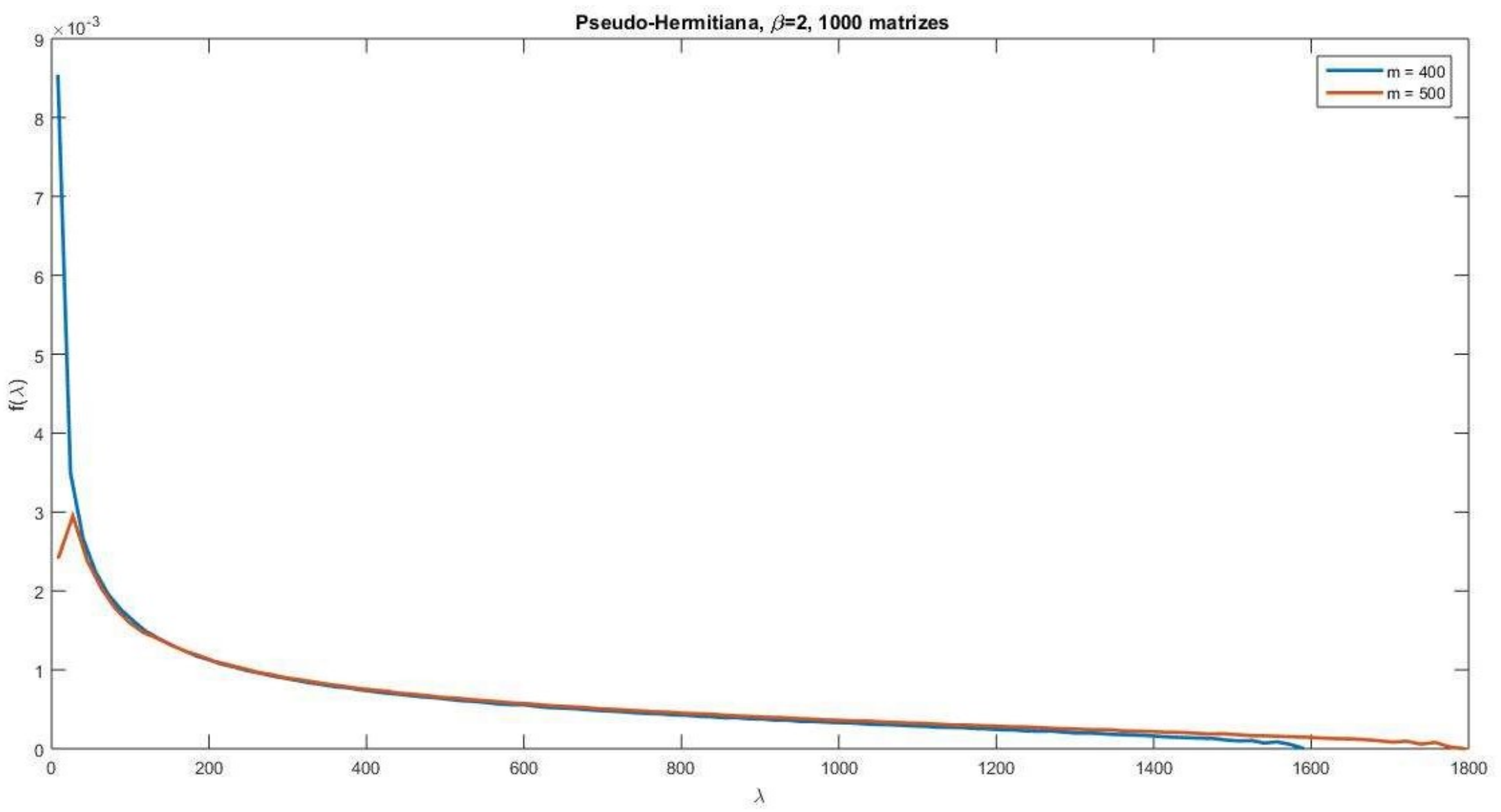

Figura 5.6: Comparação da estatística da frequência de ocorrência de autovalores para matrizes tridiagonais pseudohermitianas $(\mathrm{n}=400 \mathrm{~m}=400,500 \beta=2)$. 


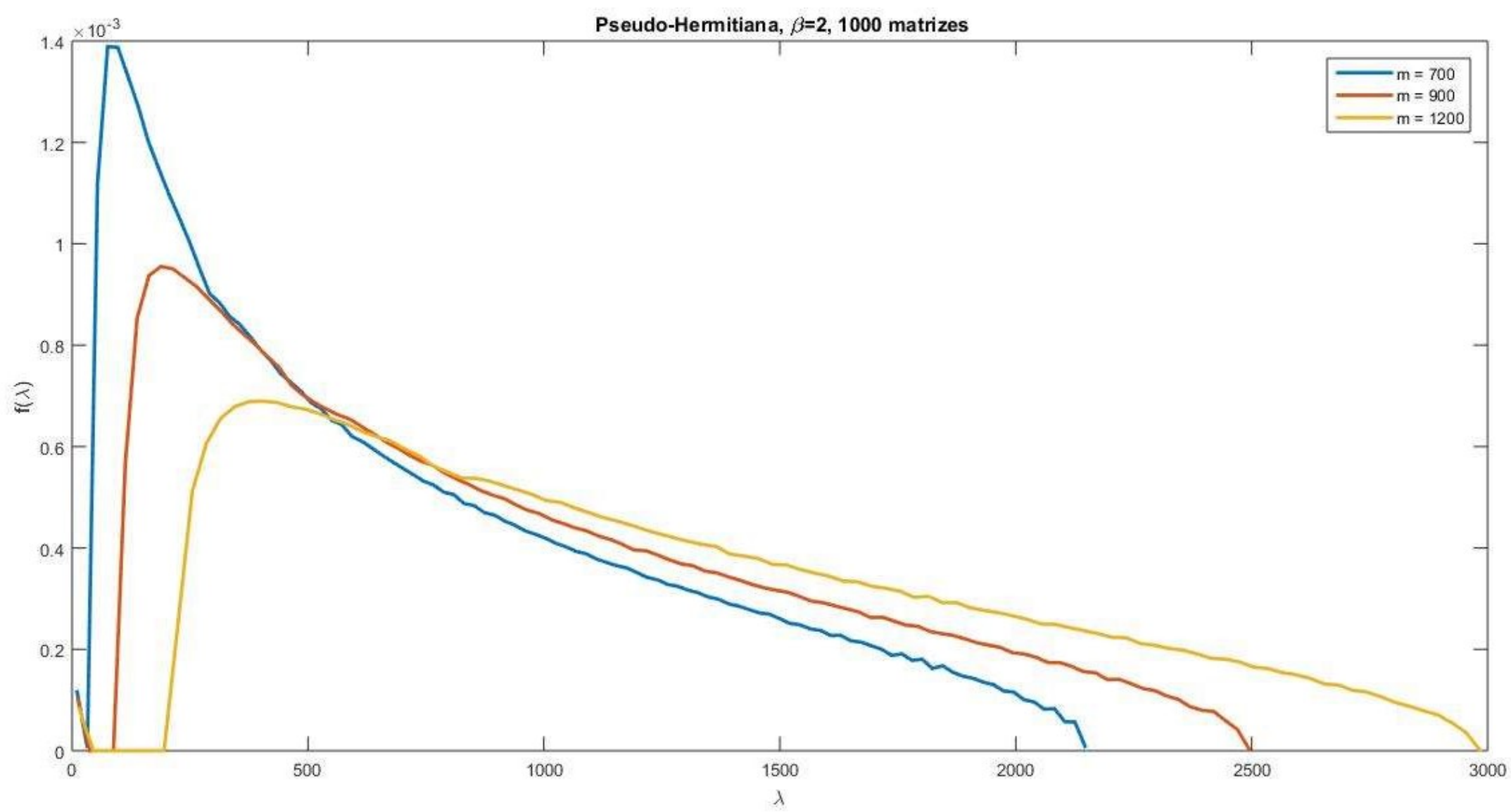

Figura 5.7: Comparação da estatística da frequência de ocorrência de autovalores para matrizes tridiagonais pseudohermitianas $(\mathrm{n}=400 \mathrm{~m}=700,900,1200 \beta=2)$. 
$n=5 \mathrm{~m}=20, \beta=2,4000$ matrizes

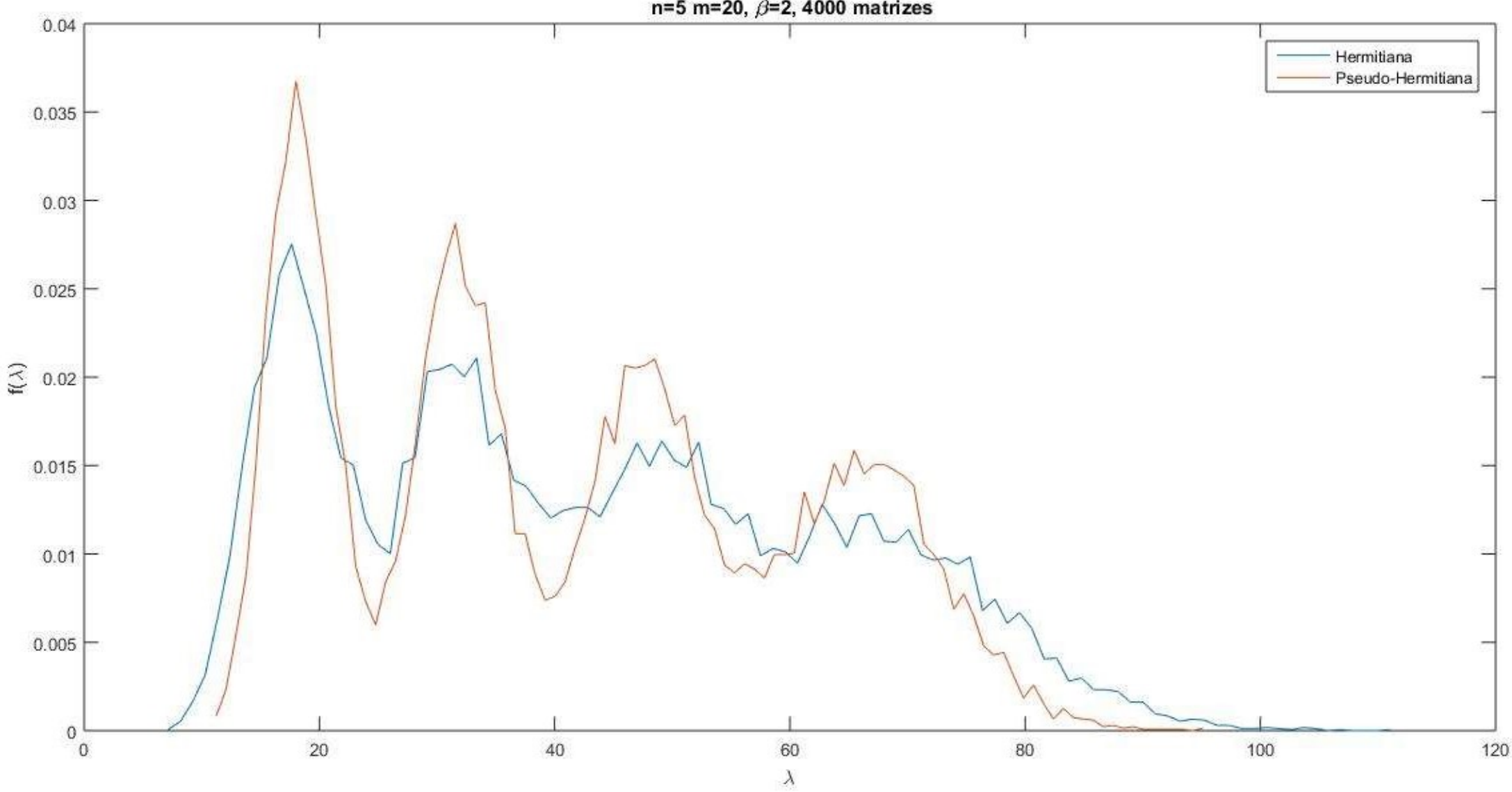

Figura 5.8: Comparação da estatística da frequência de ocorrência de autovalores para matrizes tridiagonais hermitianas e pseudo-hermitianas $(\mathrm{n}=5 \mathrm{~m}=20 \beta=2)$. 


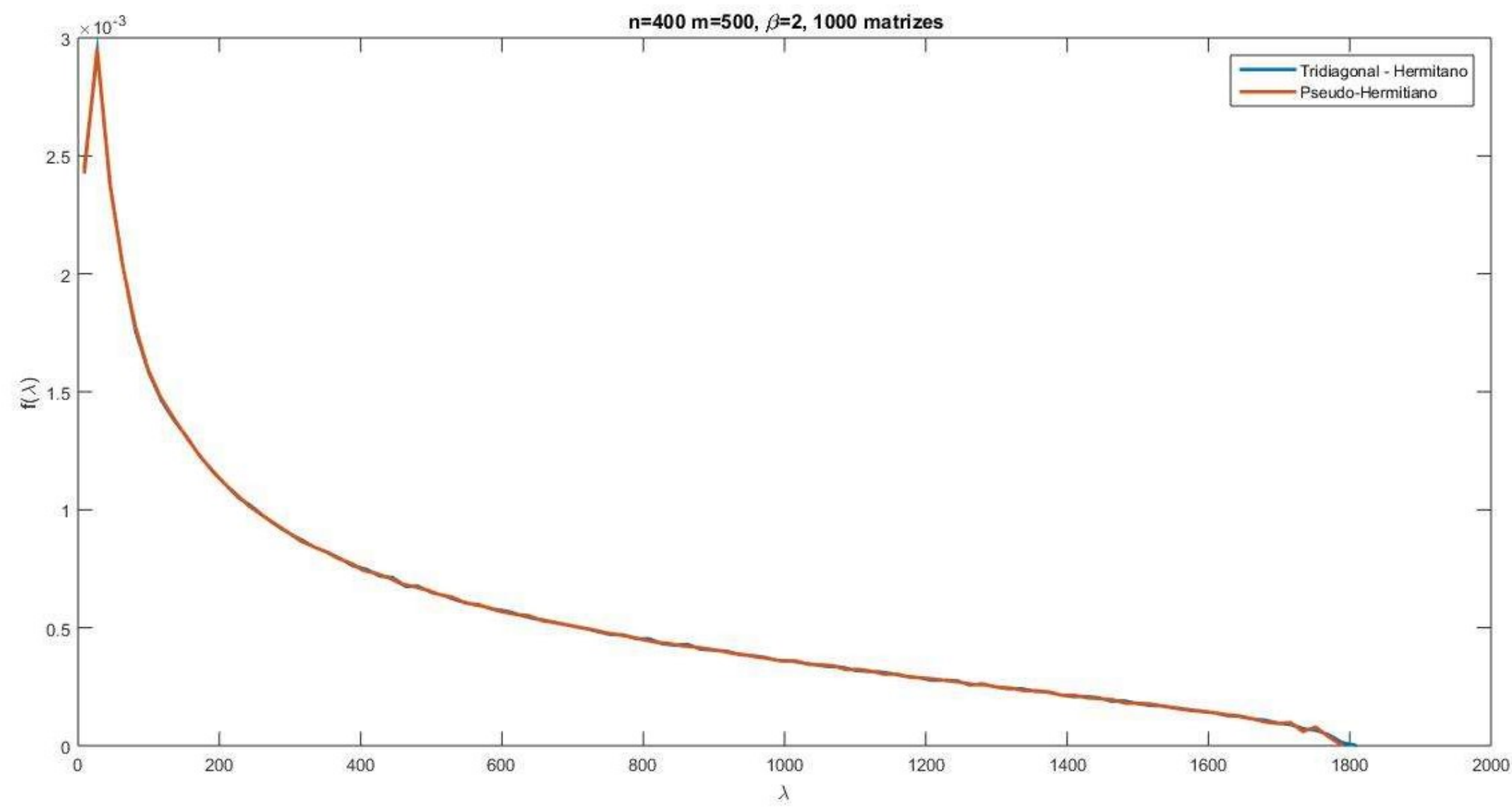

Figura 5.9: Comparação da estatística da frequência de ocorrência de autovalores para matrizes tridiagonais hermitianas e pseudo-hermitianas $(\mathrm{n}=400 \mathrm{~m}=500 \beta=2)$. 


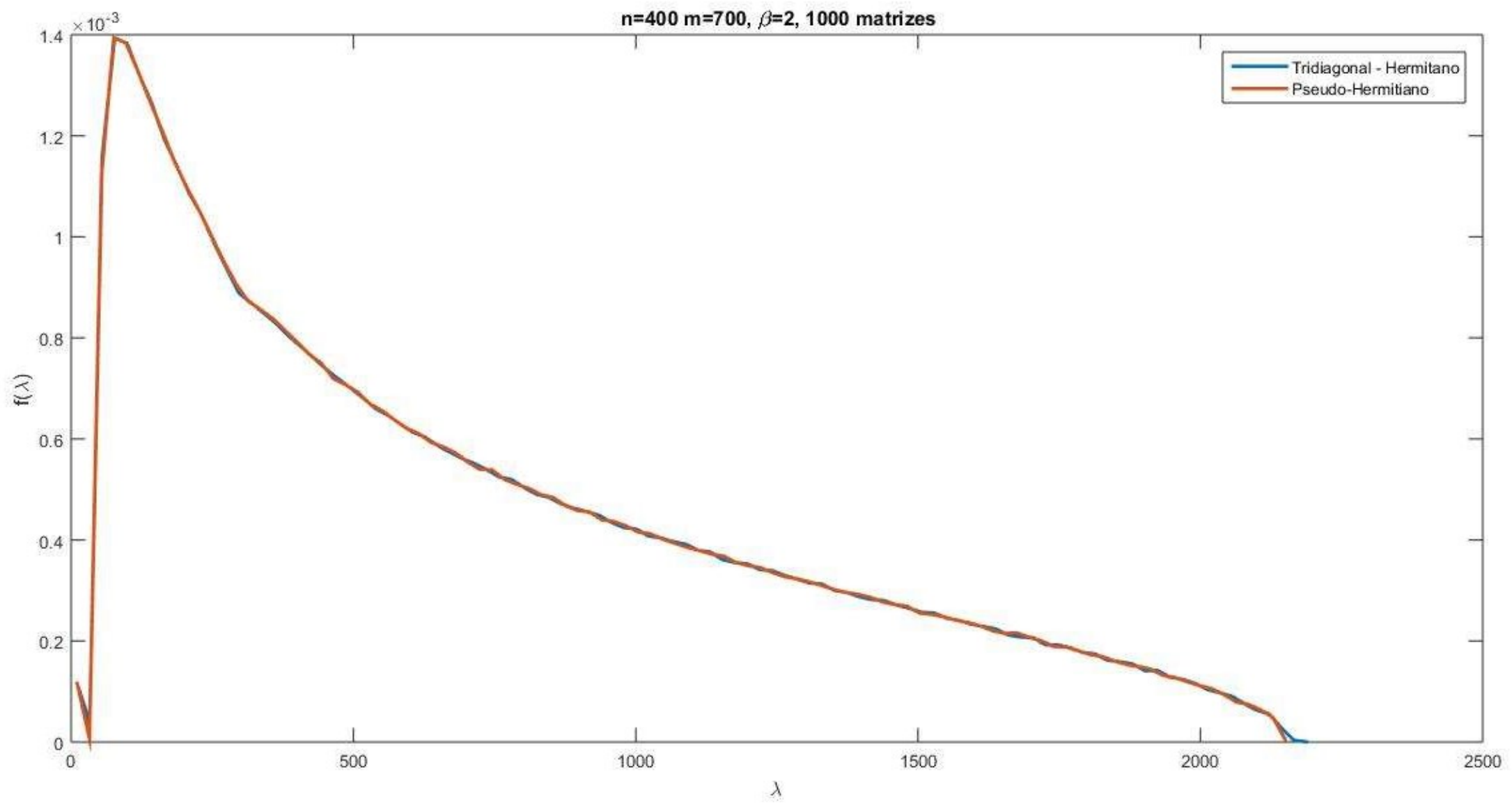

Figura 5.10: Comparação da estatística da frequência de ocorrência de autovalores para matrizes tridiagonais hermitianas e pseudo-hermitianas ( $n=400 m=700 \beta=2)$. 


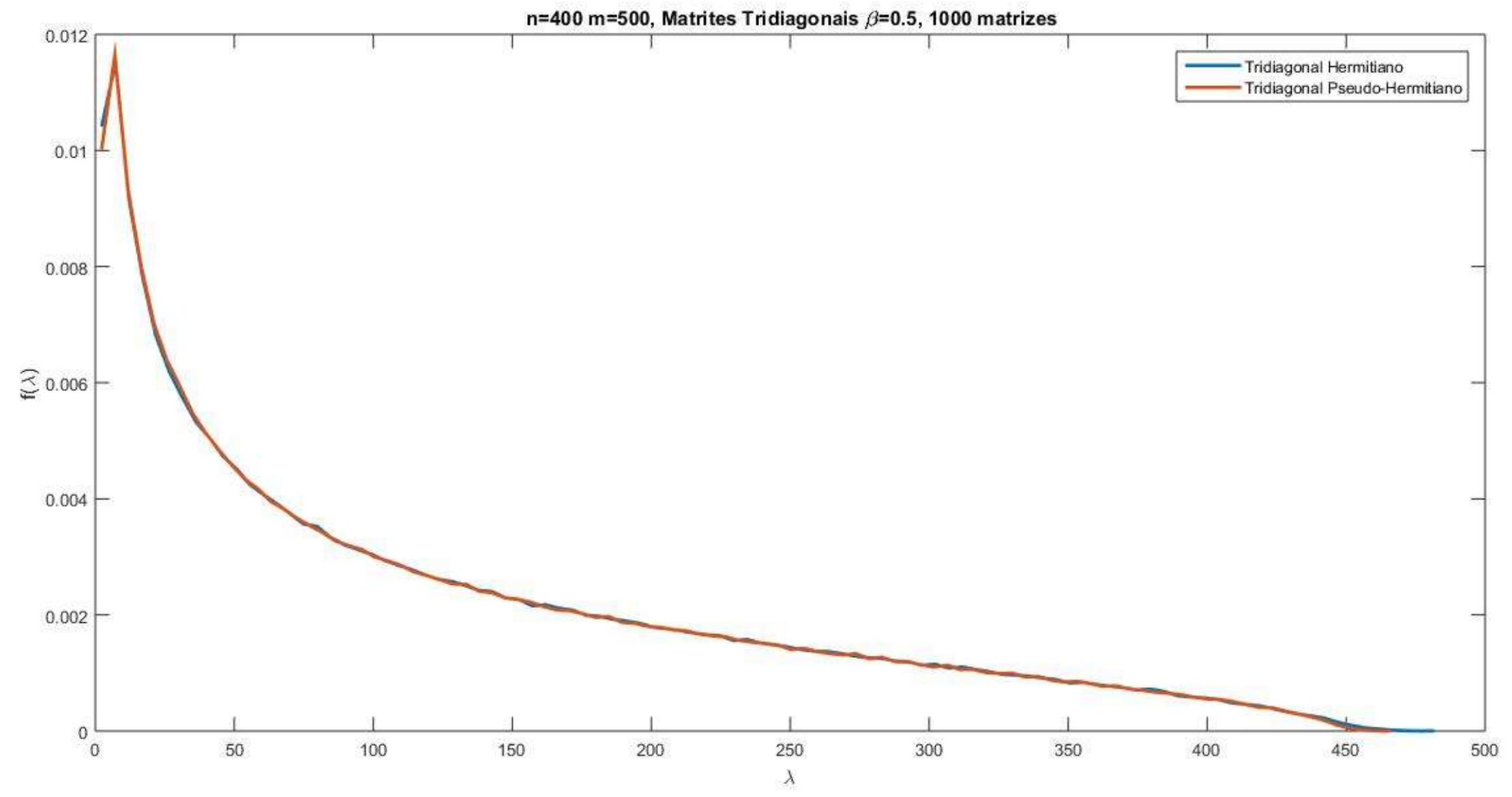

Figura 5.11: Comparação da estatística da frequência de ocorrência de autovalores para matrizes tridiagonais hermitianas e pseudo-hermitianas $(n=400 m=500 \beta=0.5)$. 


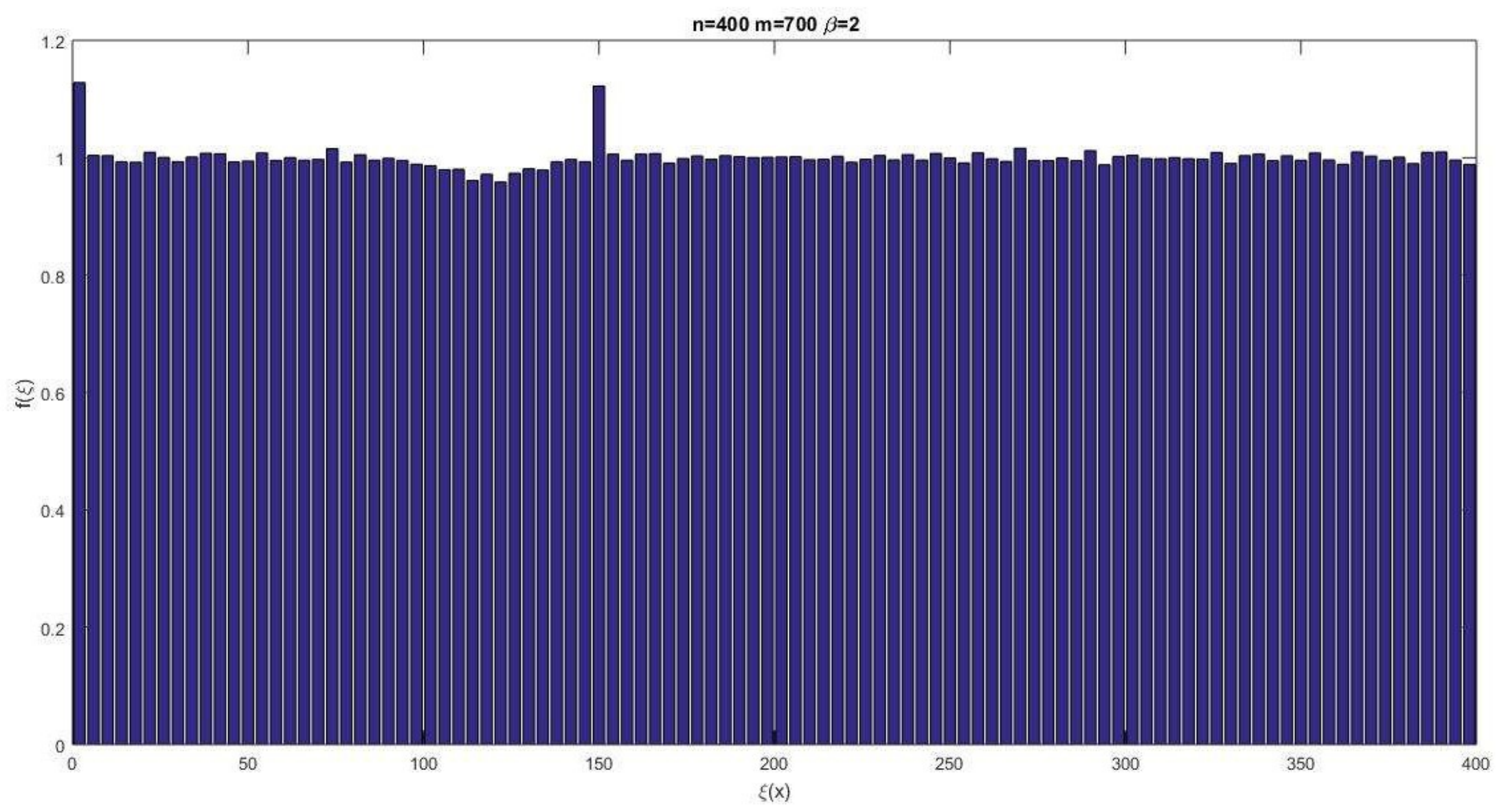

Figura 5.12: Unfolding de matrizes tridiagonais hermitianas $(\mathrm{n}=400 \mathrm{~m}=700 \beta=2)$. 


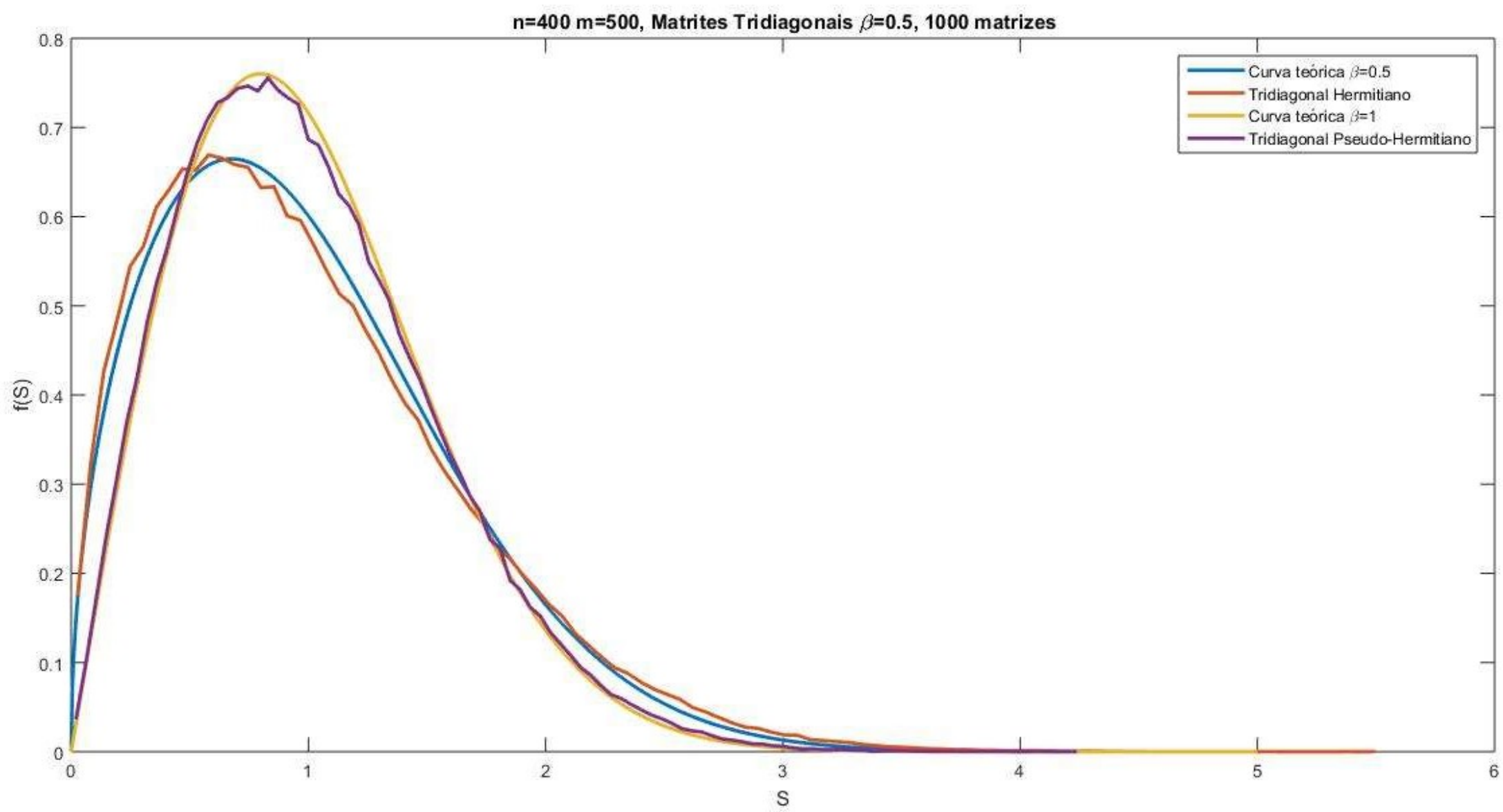

Figura 5.13: Comparação da estatística da frequência de ocorrência do espaçamento de autovalores para matrizes tridiagonais hermitianas e pseudo-hermitianas $(\mathrm{n}=400 \mathrm{~m}=500 \beta=0.5)$. 


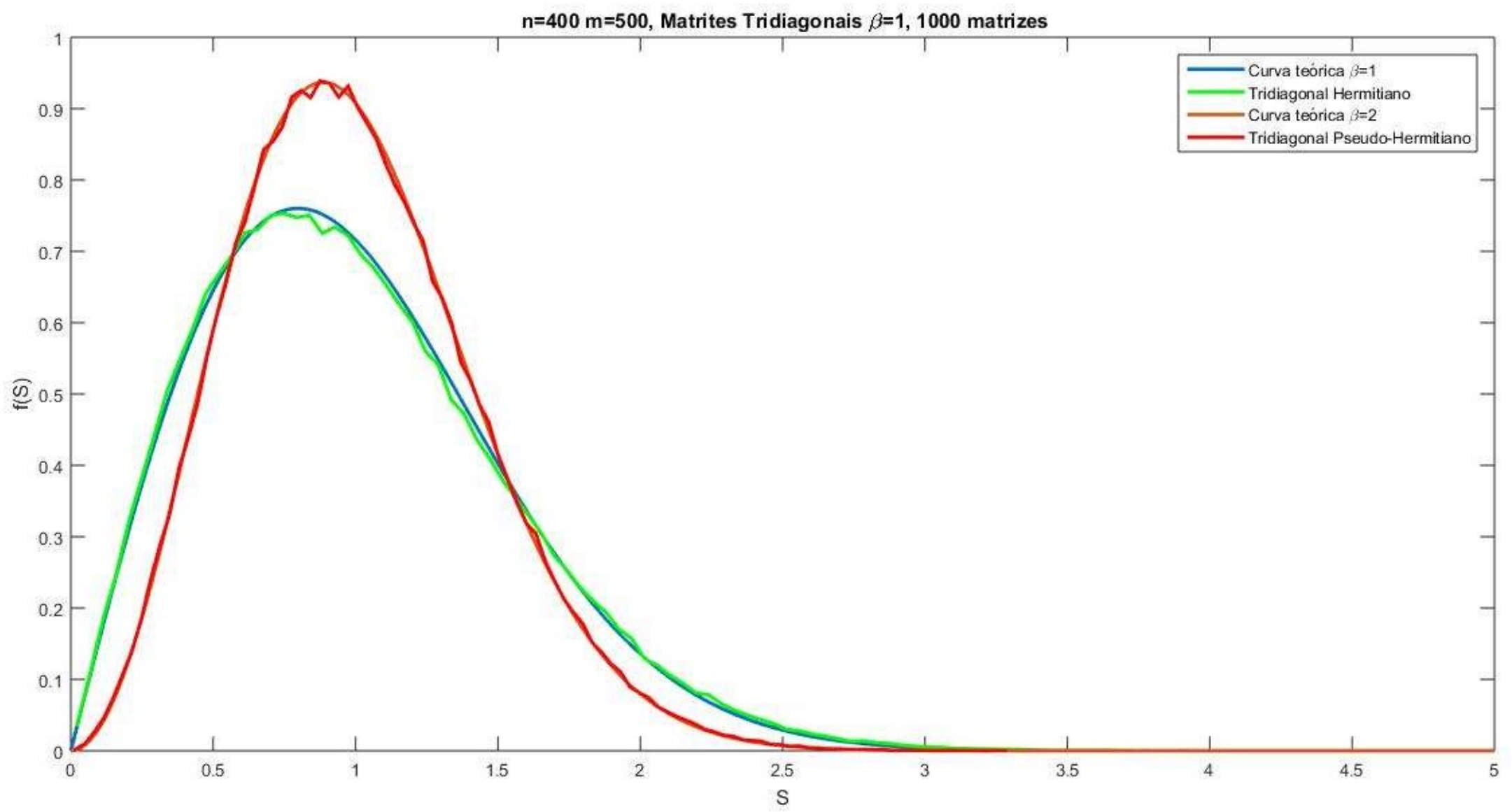

Figura 5.14 : Comparação da estatística da frequência de ocorrência do espaçamento de autovalores para matrizes tridiagonais hermitianas e pseudo-hermitianas $(\mathrm{n}=400 \mathrm{~m}=500 \beta=1)$. 


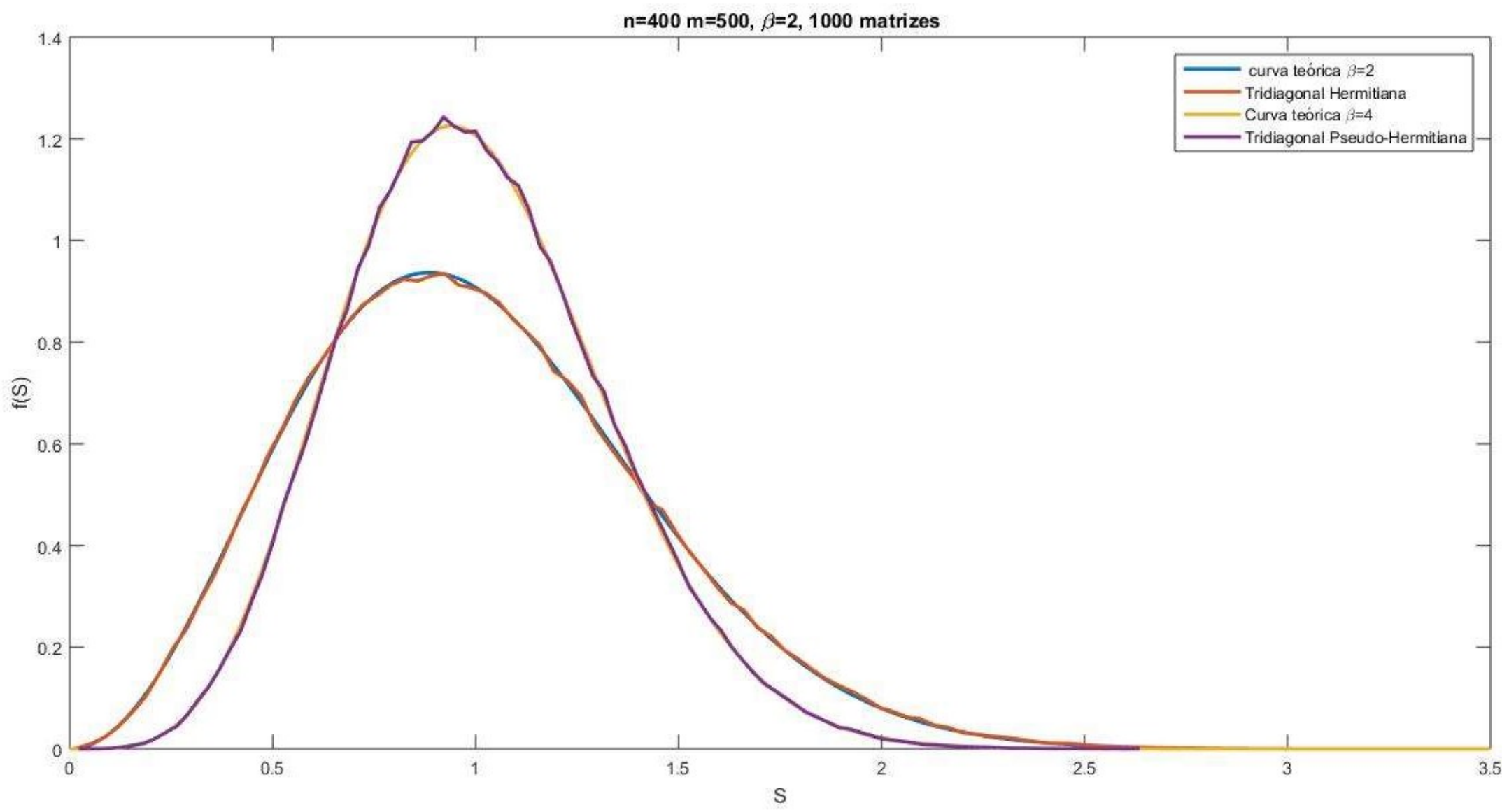

Figura 5.15: Comparação da estatística da frequência de ocorrência do espaçamento de autovalores para matrizes tridiagonais hermitianas $(\mathrm{n}=400 \mathrm{~m}=500 \beta=4)$ e pseudo-hermitianas $(\mathrm{n}=400 \mathrm{~m}=500 \beta=2)$. 


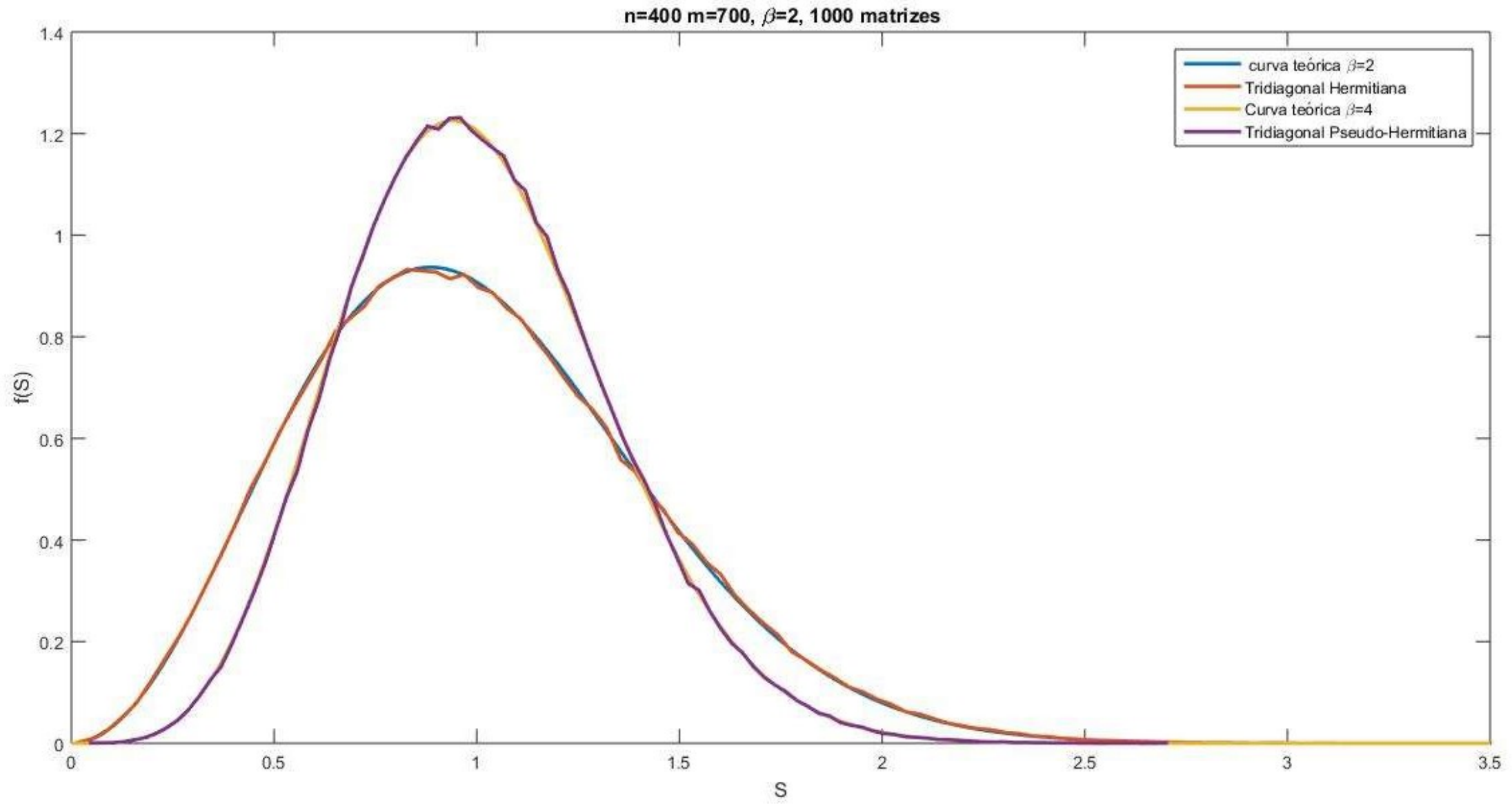

Figura 5.16: Comparação da estatística da frequência de ocorrência do espaçamento de autovalores para matrizes tridiagonais hermitianas e pseudo-hermitianas $(\mathrm{n}=400 \mathrm{~m}=700 \beta=2)$. 


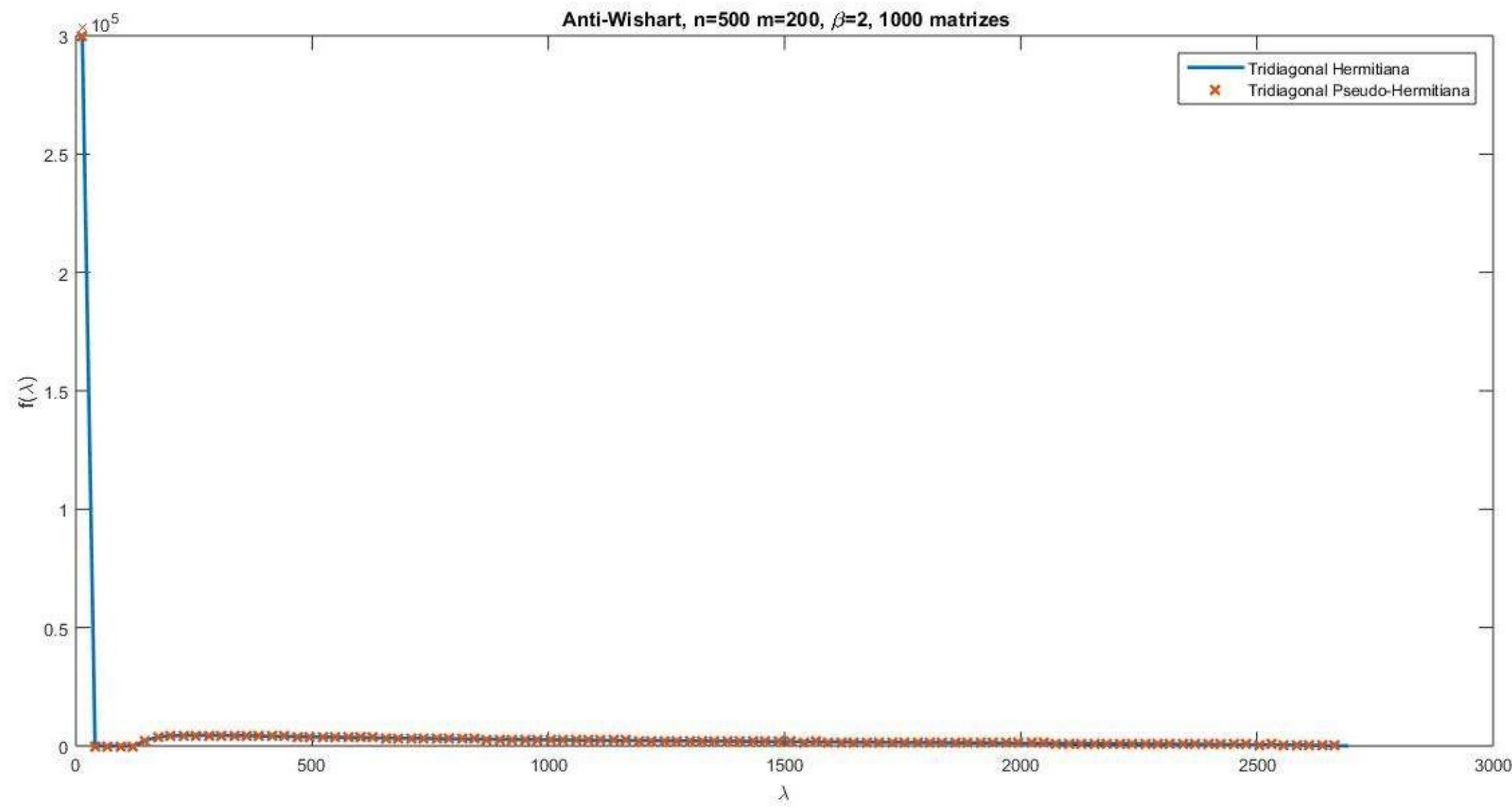

Figura 5.17: Estatística da frequência de ocorrência de autovalores para matrizes tridiagonais hermitianas e pseudo-hermitianas no ensemble anti-Wishart $W=H \cdot H^{T}(\mathrm{n}=500 \mathrm{~m}=200 \beta=2)$. 


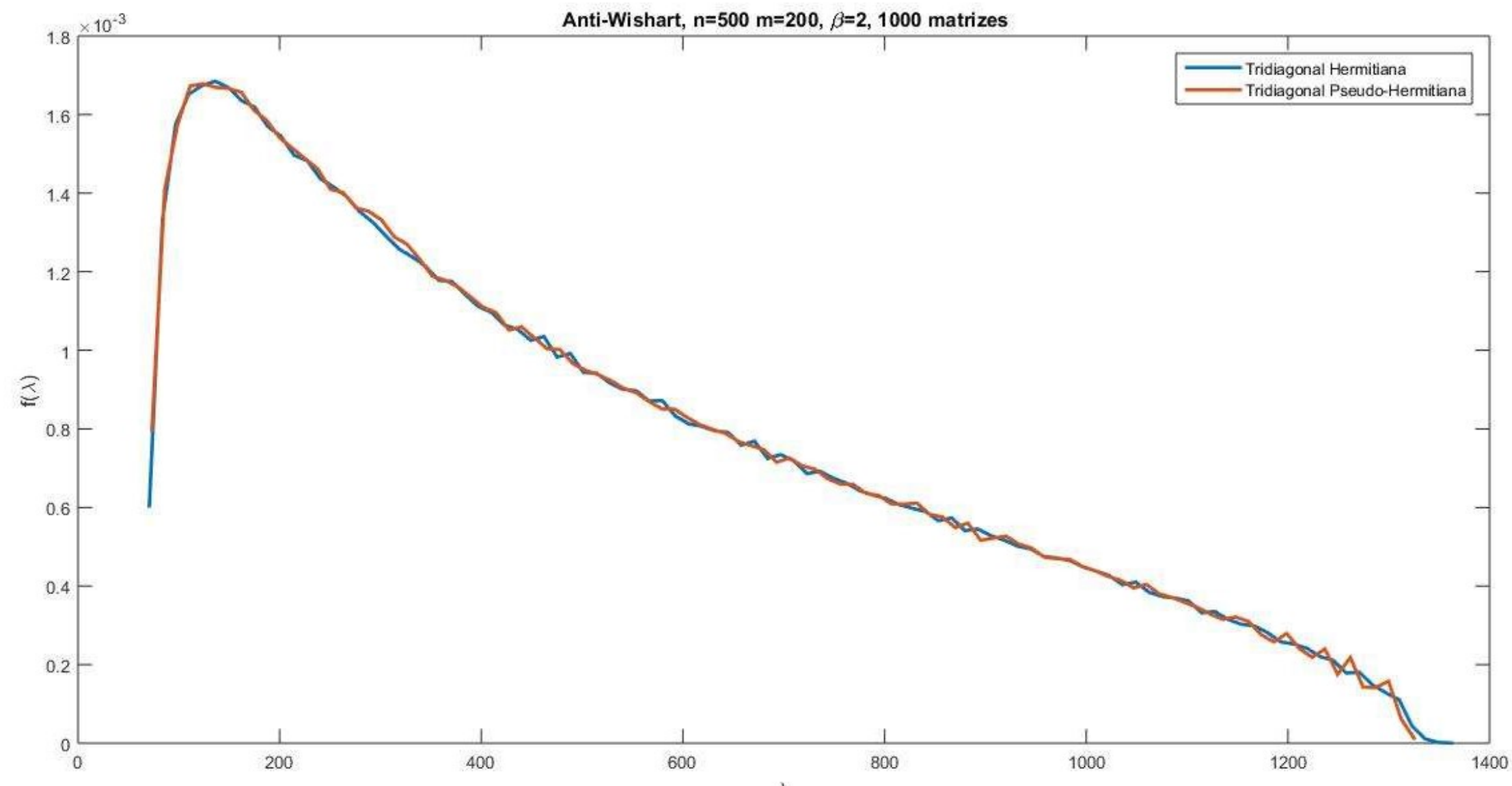

Figura 5.18: Estatística da frequência de ocorrência de autovalores para matrizes tridiagonais hermitianas e pseudo hermitanas no ensemble anti-Wishart $\tilde{W}=H^{T} \cdot H \quad(\mathrm{n}=500 \mathrm{~m}=200 \beta=2)$. 


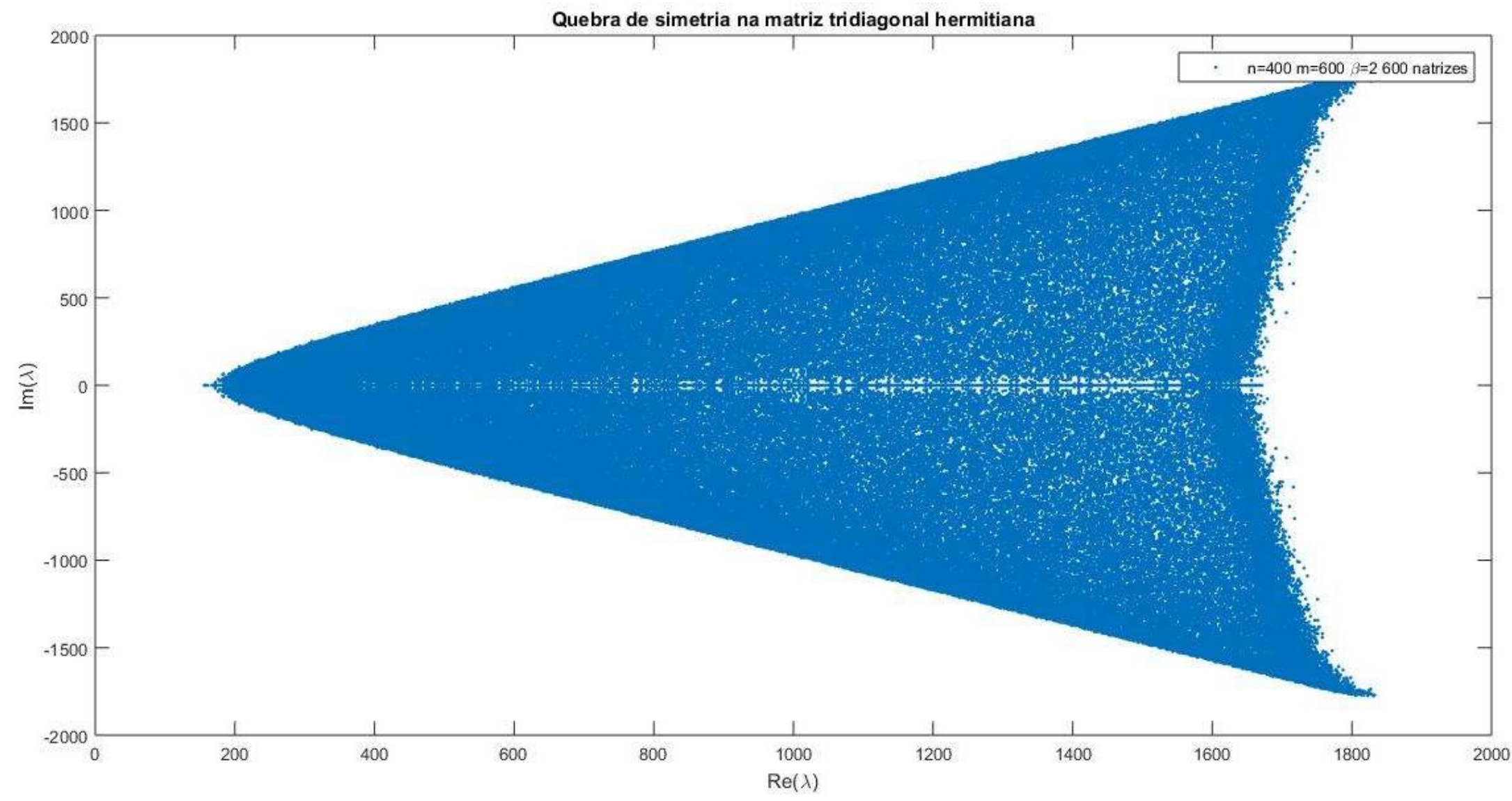

Figura 19: Estatística da frequência de ocorrência de autovalores para quebra de hermiticidade em matrizes tridiagonais hermitianas $(\mathrm{n}=400 \mathrm{~m}=500 \beta=2)$. 


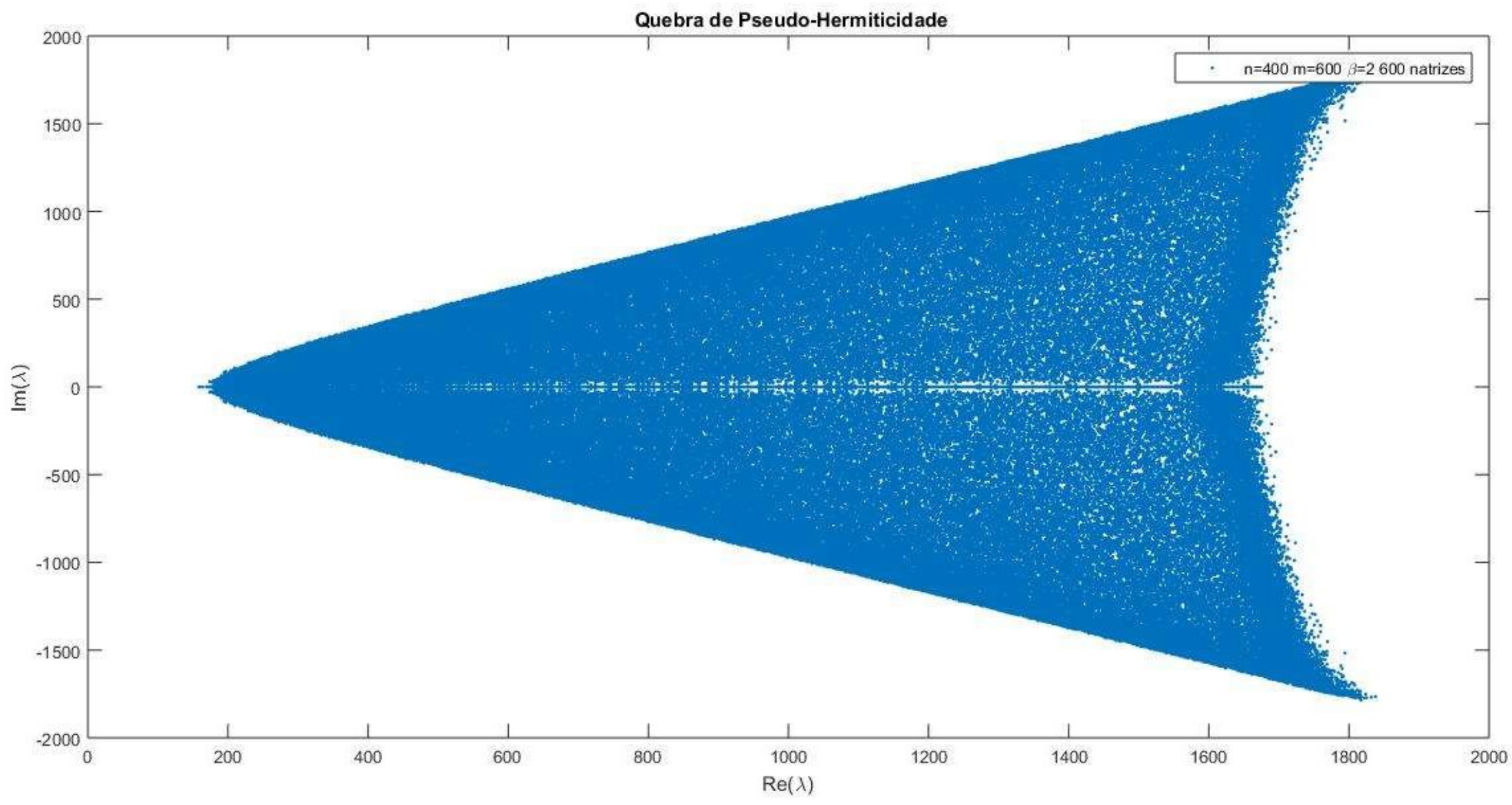

Figura 5.20: Estatística da frequência de ocorrência de autovalores para quebra de pseudo-hermiticidade $(n=400 m=500 \beta=2)$. 


\section{Conclusão}

Na década de 30, Wishart introduziu as matrizes aleatórias em um artigo sobre análise estatística multivariada de dados [12]. Na década de 50, com Wigner, essa teoria (RMT) encontraria a primeira aplicação direta em física [33], no estudo dos níveis de energia de núcleos atômicos pesados, que possuem funções de ondas complicadas. Desde então, o número de aplicações em física só aumentou, encontrando aplicações na mais diversas áreas da física. Neste trabalho, foram revisadas as principais propriedades estatísticas do ensemble clássico de Wishart, também conhecido como $\beta$-Laguerre ensemble. Como parte da originalidade do trabalho, buscou-se avaliar a estatística de matrizes tridiagonais pseudo-hermitianas que pertençam a esse ensemble.

Como ponto de partida, é apresentado no capítulo 2 a construção clássica de um elemento que pertença a esse ensemble. Essa construção é via matrizes retangulares com elementos identicamente e independentemente distribuídos com entradas gaussianas. Buscou-se então, a partir disso, a distribuição conjunta de probabilidades das entradas da matriz. Em RMT essas entradas podem ser reais, complexas ou quarteniônicas, conhecido como threefold way de Dyson, caracterizado pelo índice $\beta$ que pode assumir três valores $\beta=1,2,4$. Esse, basicamente conta o número de gaussianas reais em cada entrada. Devido a existência de trabalhos discutindo o efeito desse índice sobre a estatística do ensemble, inclusive para outros valores de $\beta$, durante a construção teórica dos modelos foi considerado apenas o caso $\beta=2$ , sendo os demais índices, mais facilmente ou não, generalizados de maneira similar. Uma discussão sobre o efeito deste índice pode ser encontrada nas referências [6,22]. Uma análise numérica foi feita para valores de $\beta$ fora do threefold way de Dyson, a fim de melhor compreender a distribuição de frequências dos espaçamentos dos autovalores nos casos hermitianos e pseudo-hermitianos.

A estatística dos autovalores é deduzida no capítulo 3. Inicialmente buscou-se uma expressão que determina o perfil do histograma obtido para qualquer valor de $n, m$, assim como o limite assintótico, $n, m \rightarrow \infty$, conhecido em literatura como distribuição de Marchenko-Pastur. Esses capítulos iniciais são importantes pois configuram a base da teoria. Eles são usados como modelos para discutir os casos tridiagonais hermitianos e tridiagonais pseudo-hermitianos.

Seguindo o procedimento realizado por Dumitriu e Edelman [13,22] é construído o modelo das matrizes tridiagonais do ensemble de Wishart no capítulo 4. Esse modelo foi divido em dois casos: 
matrizes tridiagonais hermitianas e pseudo.hermitianas. A partir dessa construção foi possível estudar a estatística dos autovalores.

A fim de melhor observar os resultados relevantes a esse ensemble, foram realizados cálculos numéricos no software $M_{a t l a b}{ }^{\circledR}$ e o resultado apresentado nas figuras disponíveis no capítulo 5 . Observou-se que a distribuição dos autovalores, para os casos hermitianos e pseudo-hermitianos, estão em acordo com o modelo clássico das matrizes cheias. Para os cálculos realizados com as matrizes tridiagonais verificou-se que o tempo de processamento é maior quando comparado a investigações similares com matrizes cheias. Observamos numericamente a precisão com que as curvas teóricas obtidas nos capítulos precedentes ajustam os histogramas gerados.

Entretanto, foram observadas diferenças para o espaçamento dos autovalores. Para o caso pseudohermitiano o valor de $\beta$ que deve ser utilizado na curva de ajuste do espaçamento, apresentada no capítulo 5 , é dada pela relação $\beta_{\text {Pseudo-Hermitiano }}=2 \cdot \beta_{\text {Hermitiano }}$. Isso é uma evidência de um comportamento observado no ensemble de Hermite devido a distribuição dos elementos pseudo-hermitianos [29]. Nesse ensemble, a distribuição estatística dos elementos no limite assintótico tende ao caso hermitiano com a correção no índice beta mencionado anteriormente.

Foi verificado que a convergência para o limite assintótico $n, m \rightarrow \infty$, tal que $\frac{n}{m} \leq 1$, é facilmente obtido. O caso $n=30 \mathrm{~m}=80$ já apresenta o perfil da distribuição de Marckenko-Pastur.

Este trabalho oferece uma revisão do clássico ensemble de Wishart, assim como uma investigação, o mais abrangente possível, de sistemas pseudo-hermitianos no referido ensemble. Este estudo é importante, pois permite o uso de técnicas desenvolvidas em RMT no estudo de hamiltonianas não convencionais que aparem em determinados sistemas quânticos [3]. O ensemble de Wishart possui alto potencial de aplicação em diversas áreas da física, como trabalhado ao longo do texto. Devido a fácil implementação, do ponto de vista computacional, torna-se uma ferramenta útil na simulação de propriedades gerais de sistemas físicos [6,7,14,16,11].

Investigações adicionais, a serem desenvolvidas em trabalhos futuros, podem ter como ponto de partida o ferramental aqui revisado e desenvolvido. Uma melhor compreensão do espaçamento de sistemas pseudo-hermtianos, do ponto de vista analítico, e uma compreensão da distribuição de autovalores no plano complexo, com a quebra de hermiticidade, são dois exemplos. 


\section{Apêndice A}

\section{Elementos de Álgebra Linear}

\section{A.1 Espaços Vetoriais}

Para operar e entender a natureza dos objetos encontrados na teoria quântica é feita uma breve introdução ao estudo de espaços vetoriais. As definições apresentadas aqui seguem o desenvolvimento de livros clássicos de álgebra linear [34].

Definição 1: Dado um conjunto $V$, cujos elementos chamamos de vetores, e um corpo $K^{3}$, dizemos que $V$ é um espaço vetorial sobre $K$ se existem duas operações

$$
(\text { soma })+: V \times V \rightarrow V
$$

e

$$
\text { (produto) *: } V \times V \rightarrow V
$$

tal que para todo $\left|\mathrm{v}_{i}\right\rangle \in V$ e $\alpha, \beta \in K$ as seguintes propriedades são verificadas,

1) Comutatividade: $\left|\mathrm{v}_{1}\right\rangle+\left|\mathrm{v}_{2}\right\rangle=\left|\mathrm{v}_{2}\right\rangle+\left|\mathrm{v}_{1}\right\rangle$

2) Associatividade: $\left(\left|\mathrm{v}_{1}\right\rangle+\left|\mathrm{v}_{2}\right\rangle\right)+\left|\mathrm{v}_{3}\right\rangle=\left|\mathrm{v}_{1}\right\rangle+\left(\left|\mathrm{v}_{2}\right\rangle+\left|\mathrm{v}_{3}\right\rangle\right)$ e $\alpha\left(\beta\left|\mathrm{v}_{i}\right\rangle\right)=(\alpha \beta)\left|\mathrm{v}_{i}\right\rangle$

3) Distributividade: $\alpha\left(\left|\mathrm{v}_{1}\right\rangle+\left|\mathrm{v}_{2}\right\rangle\right)=\alpha\left|\mathrm{v}_{1}\right\rangle+\alpha\left|\mathrm{v}_{2}\right\rangle$ e $(\alpha+\beta)\left|\mathrm{v}_{i}\right\rangle=\alpha\left|\mathrm{v}_{i}\right\rangle+\beta\left|\mathrm{v}_{i}\right\rangle$

${ }^{3} \mathbb{R}$ (reais) ou $\mathbb{C}$ (complexos), por exemplo. 
4) Elemento neutro para a adição: $\left|\mathrm{v}_{i}\right\rangle+|0\rangle=\left|\mathrm{v}_{i}\right\rangle$

5) Inverso aditivo: $\left|\mathrm{v}_{i}\right\rangle+\left|-\mathrm{v}_{i}\right\rangle=|0\rangle$

6) Elemento neutro para a multiplicação: $|1\rangle *\left|\mathrm{v}_{i}\right\rangle=\left|\mathrm{v}_{i}\right\rangle$.

Definição 2: Seja $W$ um subconjunto não vazio de $V$,que também seja espaço vetorial, nessas condições $W$ é denominado subespaço vetorial de $V$.

Definição 3: Dado um subconjunto $X \subseteq V$, o conjunto das combinações lineares dos vetores de $X$, isto é,

$$
\left.C(X)=\left\{\sum_{i=1}^{n} \alpha_{i}\left|\mathrm{v}_{i}\right\rangle\left|\alpha_{i} \in K,\right| \mathrm{v}_{i}\right\rangle \in V\right\}
$$

é um subespaço de $V$, denominado subespaço gerado por $X$.

Definição 4: Dados dois espaços vetoriais, $V$ e $W$, sobre um corpo $K$ dizemos que uma função $f$,

$$
f: V \rightarrow W
$$

é linear se satisfaz a seguinte propriedade,

$$
f\left(\alpha\left|\mathrm{v}_{1}\right\rangle+\beta\left|\mathrm{v}_{2}\right\rangle\right)=\alpha f\left(\left|\mathrm{v}_{1}\right\rangle\right)+\beta f\left|\mathrm{v}_{2}\right\rangle
$$

para quaisquer $\left|\mathrm{v}_{i}\right\rangle \in V$ e $\alpha_{i} \in K$.

Na situação em que os espaços vetoriais $V$ e $W$ coincidem, chamamos a função $f$ de operador linear. 
A fim de definirmos o produto interno é importante introduzir um outro espaço vetorial denominado de espaço vetorial dual. Este, é construído de maneira unívoca a partir de $V$, de tal maneira que para todo elemento em $V$ associamos um único elemento no seu dual $\left(V^{*}\right)$, ou seja,

$$
\left\langle\mathrm{v}_{i}|\longleftrightarrow| \mathrm{v}_{i}\right\rangle
$$

$\operatorname{com}\left|\mathrm{v}_{i}\right\rangle \in V$ e $\left\langle\mathrm{v}_{i}\right| \in V^{*}{ }^{4}$. A fim de evitar maiores complicações, que fogem ao escopo do texto, postulase que dado um espaço vetorial sobre o corpo dos complexos a seguinte relação é verificada no caso dual

$$
\alpha\left|\mathrm{v}_{1}\right\rangle+\beta\left|\mathrm{v}_{2}\right\rangle \underset{C D}{\longleftrightarrow} \alpha^{*}\left\langle\mathrm{v}_{1}\right|+\beta^{*}\left\langle\mathrm{v}_{2}\right|
$$

onde $C D$ e * denotam, respectivamente, correspondência dual e conjugado complexo. Um bra pode ser entendido como uma função linear atuando sobre um determinado espaço vetorial, de tal forma que o conjunto de todas as funções lineares atuando sobre vetores neste espaço vetorial, $\langle\bullet|=f: V \rightarrow K$, seja denominada espaço vetorial dual.

Definição 5: Dado um espaço vetorial $V$, sobre o corpo dos complexos, definimos o produto interno como uma função

$$
\langle\bullet \mid \bullet\rangle: V \times V \rightarrow \mathbb{C}
$$

que satisfaz as propriedades:

- $\left\langle\mathrm{v}_{1} \mid \mathrm{v}_{2}\right\rangle=\left\langle\mathrm{v}_{2} \mid \mathrm{v}_{1}\right\rangle^{*}$

- $\left\langle\mathrm{v}_{1} \mid \alpha \mathrm{v}_{2}+\beta \mathrm{v}_{3}\right\rangle=\alpha\left\langle\mathrm{v}_{1} \mid \mathrm{v}_{2}\right\rangle+\beta\left\langle\mathrm{v}_{1} \mid \mathrm{v}_{3}\right\rangle$, para qualquer $\left|\mathrm{v}_{i}\right\rangle \in V$ e $\alpha \in \beta$.

${ }^{4}$ Em notação de Dirac o elemento 〈๑|é chamado de "bra" e $|\bullet\rangle$ de "ket". 
- $\left\langle\mathrm{v}_{i} \mid \mathrm{v}_{i}\right\rangle \geq 0$, onde a igualdade é apenas verificada para o vetor nulo.

A partir das propriedades acima, é fácil verificar que o produto interno é antilinear na primeira entrada, ou seja, $\left\langle\alpha \mathrm{v}_{1}+\beta \mathrm{v}_{2} \mid \mathrm{v}_{3}\right\rangle=\alpha^{*}\left\langle\mathrm{v}_{1} \mid \mathrm{v}_{3}\right\rangle+\beta^{*}\left\langle\mathrm{v}_{2} \mid \mathrm{v}_{3}\right\rangle$. A verificação segue da aplicação das duas primeiras propriedades.

Definição 6: Dado um conjunto de vetores $\left\{\left|\mathrm{v}_{1}\right\rangle, \ldots,\left|\mathrm{v}_{n}\right\rangle\right\} \in V$ dizemos que eles são linearmente independentes (L.I.) se a única solução possível para a equação,

$$
\sum_{i=1}^{n} \alpha_{i}\left|\mathrm{v}_{i}\right\rangle=0
$$

é a trivial, ou seja, $\alpha_{1}=\ldots=\alpha_{n}=0$.

A definição anterior implica que nenhum dos vetores pode ser escrito como combinação linear dos demais.

Proposição 7: Dado um conjunto de vetores $\left\{\left|\mathrm{v}_{1}\right\rangle, \ldots,\left|\mathrm{v}_{n}\right\rangle\right\} \in V$ linearmente independentes e um subespaço $E$ gerado por esse conjunto. Então, cada vetor de $E$ pode ser escrito de maneira única como combinação linear dos vetores $\left\{\left|\mathrm{v}_{1}\right\rangle, \ldots,\left|\mathrm{v}_{n}\right\rangle\right\}$.

Demonstração: Por definição temos que todo vetor $|\mathrm{v}\rangle \in E$ pode ser escrito como combinação linear $\operatorname{dos}\left\{\left|\mathrm{v}_{i}\right\rangle\right\}$. Suponha por exista duas maneiras de escrever a combinação linear, ou seja,

$$
|\mathrm{v}\rangle=\sum_{i=1}^{n} \alpha_{i}\left|\mathrm{v}_{i}\right\rangle=\sum_{i=1}^{n} \beta_{i}\left|\mathrm{v}_{i}\right\rangle \Rightarrow \sum_{i=1}^{n}\left(\alpha_{i}-\beta_{i}\right)\left|\mathrm{v}_{i}\right\rangle=0
$$


utilizando o fato de os vetores serem linearmente independentes temos imediatamente que,

$$
\left(\alpha_{i}-\beta_{i}\right)=0 \Leftrightarrow \alpha_{i}=\beta_{i},
$$

implicando que a combinação linear é única.

Definição 8: Na condição acima, os vetores $\left\{\left|\mathrm{v}_{1}\right\rangle, \ldots,\left|\mathrm{v}_{n}\right\rangle\right\}$ são chamados de base para $E$.

Uma base gera todos os vetores de um determinado espaço vetorial. A dimensão do espaço corresponde ao número de elementos da base.

Como operadores lineares preservam combinações lineares, a verificação é imediata a partir da definição, normalmente trabalha-se com as componentes de um vetor em uma determinada base.

$$
|\mathrm{v}\rangle=\left[\begin{array}{c}
\mathrm{v}_{1} \\
\mathrm{v}_{2} \\
\vdots \\
\mathrm{v}_{n}
\end{array}\right] \rightarrow\langle\mathrm{v}|=\left[\begin{array}{llll}
\mathrm{v}_{1}^{*} & \mathrm{v}_{2}^{*} & \cdots & \mathrm{v}_{n}^{*}
\end{array}\right]
$$

dessa forma podemos utilizar matrizes quadradas para representar operadores lineares.

Definição 9: Dado um operador linear $T$, que em uma determinada base possui representação matricial da forma $T_{i j}$, podemos definir a matriz que representa o operador adjunto $T^{\dagger}$ como o transposto conjugado de $T$, ou seja, $\left(T_{i j}\right)^{\dagger}=T_{j i}^{*}$. 


\section{A.2 Tópicos Matriciais}

Uma matriz é uma tabela organizada em $n$ linhas e $m$ colunas com entradas sobre um corpo $K$ . O conjunto de todas as matrizes sobre esse corpo é denotada por $M_{n m}(K)$, e no caso $n=m$ a matriz é dita quadrada. Um elemento de uma matriz $A$ normalmente é representado por $[A]_{i j}$, onde $i$ e $j$ denotam respectivamente a linha e a coluna do elemento.

Corolário 1: O conjunto de todas as matrizes $M_{n m}(K)$, com as operações usuais de adição entre matrizes e multiplicação por escalar, real ou complexo, formam um espaço vetorial.

Com um pouco de álgebra é possível verificar todas as propriedades listadas anteriormente na seção A.1. Como o resultado é facilmente encontrado em livros de álgebra linear, a verificação será suprimida.

Definição 2: Dado uma matriz $A \in M_{m n}(K)$, a transposta de $A$ denotada por $A^{T}$ é definida como a matriz $A^{T} \in M_{n m}(K)$ cujas entradas são dadas por $\left[A^{T}\right]_{i j}=[A]_{j i}$.

Naturalmente $\left(A^{T}\right)^{T}=A$. Quando para uma matriz real $A$ é verificada a relação $A^{T}=A$, então essa é dita simétrica.

Se a transposição de uma matriz é seguida da operação de tomar o conjugado complexo, a matriz obtida é dita adjunta e representada por $A^{\dagger}$.

Definição 3: Uma matriz U é dita unitária se, e somente se, satisfaz a relação,

$$
U^{\dagger} U=U U^{\dagger}=I
$$


onde $I$ denota a respectiva matriz identidade.

Em outras palavras, uma matriz é dita unitária quando a inversa corresponde a adjunta. Matrizes unitárias apresentam propriedades interessantes, a saber:

Propriedade 4: Matrizes unitárias preservam normas de vetores.

Demonstração: Seja $|\psi\rangle=U|\phi\rangle$, utilizando a definição de produto interno e de matriz unitária,

$$
\langle\psi \mid \psi\rangle=\left\langle\phi\left|U^{\dagger} U\right| \phi\right\rangle=\langle\phi|I| \phi\rangle=\langle\phi \mid \phi\rangle
$$

Propriedade 5: Autovalores de matrizes unitárias possuem módulo igual a um.

Demonstração: Seja $U|\psi\rangle=\lambda|\psi\rangle$, com $\lambda \in \mathbb{C}$, utilizando as definições anteriores, temos:

$$
\left\langle\psi\left|U^{\dagger} U\right| \psi\right\rangle=\langle\psi \mid \psi\rangle=\left\langle\psi\left|\lambda^{*} \lambda\right| \psi\right\rangle=|\lambda|^{2}\langle\psi \mid \psi\rangle
$$

como a expressão anterior deve ser verificada para qualquer vetor $|\psi\rangle$ segue imediatamente que $|\lambda|^{2}=1$

O resultado anterior pode ser interpretado de uma outra maneira, ela indica que todo autovalor de uma matriz unitária tem a forma $\lambda=e^{i \alpha}, \alpha \in \mathbb{R}$.

Propriedade 6: Autovetores de matrizes unitárias, que correspondem a autovalores distintos, são ortogonais. 
Demonstração: Considere dois autovetores de uma matriz unitária $U$, tal que, os autovalores sejam diferentes, ou seja,

$$
\left\{\begin{array}{l}
U|\psi\rangle=e^{i \alpha}|\psi\rangle \\
U|\phi\rangle=e^{i \beta}|\phi\rangle
\end{array},\right.
$$

segue então,

$$
\langle\phi \mid \psi\rangle=\left\langle\phi\left|e^{-i \beta} \cdot e^{i \alpha}\right| \psi\right\rangle=e^{i(\alpha-\beta)}\langle\phi \mid \psi\rangle
$$

o que implica,

$$
\left(e^{i(\alpha-\beta)}-1\right)\langle\phi \mid \psi\rangle=0 \rightarrow\langle\phi \mid \psi\rangle=0
$$

Definição 7: Dada uma matriz quadrada $A \in M_{n n}(K)$, um escalar $\lambda \in \mathbb{C}$ e um vetor não nulo $|\psi\rangle$, satisfazendo a equação,

$$
A|\psi\rangle=\lambda|\psi\rangle
$$

então $\lambda$ é denominado autovalor de $A$ com o respectivo autovetor $|\psi\rangle$.

Proposição 8: Considere uma transformação unitária, ou seja, satisfazendo a definição 3. Os $n$ autovalores da matriz $U A U^{-1}$ são os mesmos da matriz $\mathrm{A}$ e os autovetores são dados por $U\left|\psi_{k}\right\rangle, k \in n$. 
Demonstração: Seja $\lambda_{k}$ um dos autovalores da matriz $A$ associado ao respectivo autovetor $\left|\psi_{k}\right\rangle$. Utilizando a definição 3 e 7, temos:

$$
A\left|\psi_{k}\right\rangle=\lambda_{k}\left|\psi_{k}\right\rangle \Leftrightarrow A(\underbrace{U^{-1} U}_{I})\left|\psi_{k}\right\rangle=\lambda_{k}\left|\psi_{k}\right\rangle \Leftrightarrow\left(U A U^{1}\right) U\left|\psi_{k}\right\rangle=\lambda_{k} U\left|\psi_{k}\right\rangle,
$$

o que concluí a prova.

A fim de completar a discussão serão apresentados a seguir dois importantes teoremas relacionados a matrizes unitárias, onde, a prova pode ser facilmente consultada em literatura de análise matricial [35].

Teorema 9 (Teorema espectral): Dada uma matriz unitária $A$, existe uma matriz unitária $U$ e uma matriz diagonal $D$, tal que,

$$
A=U D U^{-1}
$$

Teorema 10 (Decomposição em valores singulares ${ }^{5}$ ): Seja uma matriz $A \in M_{n m}(\mathbb{C}$ ), então $A$ pode ser fatorada em,

$$
A=U \Sigma V^{\dagger}
$$

onde $U \in M_{n n}(\mathbb{C})$ e $V \in M_{m m}(\mathbb{C})$ são unitárias e a matriz $\Sigma \in M_{n m}(\mathbb{C})$ é diagonal.

Os elementos da matriz diagonal são denominados de valores singulares de $A$ e as colunas das matrizes $U$ e $V$ são chamados de vetores singulares a esquerda e a direita, respectivamente.

\footnotetext{
${ }^{5}$ Do inglês SVD (SIngular Value Decomposition)
} 


\section{A.3 Refletores de Householder}

Ao conjunto de transformações lineares que descrevem uma reflexão ao redor de um plano, que contém a origem, é denominado refletores de Householder. Dado um vetor unitário $u$ perpendicular a um plano e um vetor $x$, então, o subespaço ortogonal a $u$ atua como um espelho para refletir $x$, representado pela transformação $H$ [25].

Definição 1: Dado um vetor $u \in \mathbb{C}^{n}$ de norma euclidiana unitária, $\|u\|=1$, então $H=I-2 u u^{\dagger}$ é denominado refletor de Householder (ou transformação de Householder).

Na definição acima $I$ denota a matriz identidade adequada.

Teorema 2: Seja $H$ um refletor de Householder de acordo com a definição 1, então são válidas as relações,
a) $H H=I$
b) $H=H^{\dagger}$
c) $H H^{\dagger}=H^{\dagger} H=I$
d) $H z=z \Leftrightarrow u . z=0$
e) $H u=-u$

Demonstração:

a) $H H=\left(I-2 u u^{\dagger}\right)\left(I-2 u u^{\dagger}\right)=I-4 u u^{\dagger}+4 u \underbrace{u^{\dagger} u}_{\|u\|_{2}=1} u^{\dagger}=I$ 
b) $H^{\dagger}=\left(I-2 u u^{\dagger}\right)^{\dagger}=I-2\left(u u^{\dagger}\right)^{\dagger}=I-2 \underbrace{\left(u^{\dagger}\right)^{\dagger}}_{u} u^{\dagger}=H$

c) Segue imediatamente das anteriores.

d) $H z=\left(I-2 u u^{\dagger}\right) z=z-2 u \underbrace{u^{\dagger} z}_{0}=z$

e) $H z=\left(I-2 u u^{\dagger}\right) u=z-2 u \underbrace{u^{\dagger} u}_{1}=-u$

É conveniente estender a discussão para vetores $u$ não unitários. A proposição seguinte, facilmente verificada a partir do teorema anterior, garante duas propriedades de interesse de um refletor de Householder.

Proposição 3: Dado um vetor $u$, não unitário, então para $H=I-\frac{u u^{\dagger}}{\tau}$, com $\tau=\frac{\|u\|^{2}}{2}$ as relações a seguir são verificadas,
a) $H u=-u$
b) $H z=z \Leftrightarrow u . z=0$

Teorema 4: Dados dois vetores $x, y \in \mathbb{C}^{n}$ não nulos satisfazendo $\|x\|=\|y\|$ e $x \neq y$, então existe um refletor $H$ que satisfaz a relação $H x=y$.

Demonstração: Utilizando a geometria como motivação, podemos usar como ansatz que existe um vetor com a forma $u=\alpha(x-y)$ que faz com que o refletor $H$ atenda a condição do teorema anterior. Sendo 


$$
H=I-\frac{[\alpha(x-y)][\alpha(x-y)]^{\dagger}}{\tau}=I-\frac{\alpha^{2}}{\tau}\left[x^{2}-x y^{\dagger}-y x^{\dagger}+y^{2}\right],
$$

temos que a aplicação deste refletor produz,

$$
H x=x-\frac{\alpha^{2}}{\tau}\left[x^{2} x-x y^{\dagger} x-y x^{\dagger} x+y^{2} x\right]=x-\frac{\alpha^{2}}{\tau}[\underbrace{x^{2} x-x y^{\dagger} x}_{-(x-y) y^{\dagger} x} \underbrace{-y x^{\dagger} x+y^{2} x}_{(x-y) y^{2}}],
$$

reagrupando os termos,

$$
H x=x-\frac{\alpha^{2}}{\tau}\left[y^{2}-y^{\dagger} x\right](x-y),
$$

o que permite definir a constante $\alpha$, tal que,

$$
\alpha= \pm \sqrt{\frac{\tau}{y^{2}-y^{\dagger} x}} \rightarrow \frac{\alpha^{2}}{\tau}\left[y^{2}-y^{\dagger} x\right]=1
$$

produzindo, dessa forma, o resultado desejado,

$$
H x=x-(x-y)=y .
$$

O teorema anterior é importante pois garante que existe uma transformação particular que zere todas as coordenadas de um vetor, linha ou coluna, $x$, exceto uma, como segue, 


$$
H x=\left(\begin{array}{c}
x^{2} \\
0 \\
\vdots \\
0
\end{array}\right) .
$$

O procedimento é utilizado em algoritmos de álgebra linear numérica para zerar elementos, exceto um, de colunas ou linhas de matrizes [36].

\section{A.4 Determinante de Vandermonde}

Completando a discussão de álgebra linear, será feita uma breve análise envolvendo importantes resultados acerca de um determinante recorrente em teoria de matrizes aleatórias, o chamado determinante de Vandermonde. Esse determinante aparece como Jacobiano de transformações de coordenadas de elementos de uma matriz, de um dado ensemble, para os autovalores e autovetores.

Considere uma matriz tridiagonal $T$ com elementos todos positivos nas subdiagonais, escrita na forma de blocos, tal que no canto inferior direito temos uma matriz quadrada tridiagonal $T^{k}$ de tamanho $k$. Explicitamente, temos:

$$
T=\left[\begin{array}{ccccc|cccc}
a_{n} & b_{n-1} & 0 & 0 & 0 & 0 & 0 & \cdots & 0 \\
b_{n-1} & a_{n-1} & b_{n-2} & 0 & 0 & 0 & 0 & \cdots & 0 \\
0 & b_{n-2} & a_{n-2} & \ddots & 0 & 0 & 0 & \cdots & 0 \\
\vdots & \vdots & \ddots & \ddots & b_{k+1} & 0 & 0 & \cdots & 0 \\
0 & 0 & 0 & b_{k+1} & a_{k+1} & b_{k} & 0 & \cdots & 0 \\
\hline 0 & 0 & 0 & 0 & b_{k} & & & & \\
0 & 0 & 0 & 0 & 0 & & & T^{k} & \\
\vdots & \vdots & \vdots & \vdots & \vdots & & & & \\
0 & 0 & 0 & 0 & 0 & & & &
\end{array}\right],
$$


essa maneira nos permitirá trabalhar com o polinômio característico de maneira mais simples. Nas condições anteriores, podemos considerar uma decomposição da matriz $T$ em valores singulares na forma $T=Q \Sigma Q^{T}$, onde $\Sigma$ é uma matriz diagonal contendo os autovalores de $T$ e $q$ representa a primeira linha da matriz de autovetores $Q$. É conhecido em literatura que é possível obter $T$, de maneira única, partindo de $q$ e $\Sigma$ [37].

Lema 1: Para uma matriz tridiagonal com subdiagonal positiva, o determinante de Vandermonde dos autovalores ordenados é dado por,

$$
\prod_{i<j}\left(\lambda_{i}-\lambda_{j}\right)=\frac{\prod_{i=1}^{n-1} b_{i}^{i}}{\prod_{i=1}^{n} q_{i}}
$$

Demonstração: Para um dado $k$, considere que os autovalores da matriz $T^{k}$ seja representado por $\lambda_{i}^{(k)}$ , $i=1, \ldots, k$. Sabemos que esse bloco de matriz é triangular de forma que podemos escrever o polinômio característico associado a ela como,

$$
P_{k}(x)=\prod_{i=1}^{k}\left(x-\lambda_{i}^{(k)}\right),
$$

onde para esse bloco é válido a fórmula de recorrência de três termos dada por,

$$
P_{k}(x)=\left(x-a_{k}\right) P_{k-1}(x)-b_{k-1}^{2} P_{k-2}(x),
$$

com $k=1, \ldots, n$. 
A partir de (1), podemos calcular $P_{k}\left(\lambda_{j}^{k-1}\right)$ e $P_{k-1}\left(\lambda_{i}^{k}\right)$, de forma a estabelecer a seguinte igualde entre o módulo do produto dos polinômios,

$$
\prod_{\substack{1 \leq i \leq k \\ 1 \leq j \leq k-1}}\left|\lambda_{i}^{k}-\lambda_{j}^{k-1}\right|=\prod_{j=1}^{k-1}\left|P_{k}\left(\lambda_{j}^{k-1}\right)\right|=\prod_{i=1}^{k}\left|P_{k-1}\left(\lambda_{i}^{k}\right)\right|
$$

Utilizando a equação (2), e visando a relação estabelecida em (3), podemos escrever,

$$
\begin{aligned}
& \prod_{i=1}^{k-1}\left|P_{k}\left(\lambda_{i}^{k-1}\right)\right|=\prod_{i=1}^{k-1}|\left(\lambda_{i}^{k-1}-a_{k}\right) \underbrace{P_{k-1}\left(\lambda_{i}^{k-1}\right)}_{=0}|+\prod_{i=1}^{k-1}\left|b_{k-1}^{2} P_{k-2}\left(\lambda_{i}^{k-1}\right)\right| \\
& \prod_{i=1}^{k-1}\left|P_{k}\left(\lambda_{i}^{k-1}\right)\right|=b_{k-1}^{2(k-1)} \prod_{i=1}^{k-1}\left|P_{k-2}\left(\lambda_{i}^{k-1}\right)\right|
\end{aligned}
$$

que pode ser simplificada utilizando (3), de forma que,

$$
\begin{aligned}
& \prod_{i=1}^{k-1}\left|P_{k-2}\left(\lambda_{i}^{k-1}\right)\right|=\prod_{i=1}^{k-2}\left|P_{k-1}\left(\lambda_{i}^{k-2}\right)\right| \rightarrow \\
& \prod_{i=1}^{k-1}\left|P_{k}\left(\lambda_{i}^{k-1}\right)\right|=b_{k-1}^{2(k-1)} \prod_{i=1}^{k-2}\left|P_{k-1}\left(\lambda_{i}^{k-2}\right)\right|
\end{aligned}
$$

Aplicando novamente (4) e (3), e assim recorrentemente, temos: 


$$
\begin{aligned}
\prod_{i=1}^{k-1}\left|P_{k}\left(\lambda_{i}^{k-1}\right)\right| & =b_{k-1}^{2(k-1)} \cdot b_{k-2}^{2(k-2)} \prod_{i=1}^{k-3}\left|P_{k-2}\left(\lambda_{i}^{k-3}\right)\right| \\
& =\cdots \\
& =\prod_{i=1}^{n-1} b_{i}^{2 i}
\end{aligned}
$$

Para completar a prova é necessário recorrer ao teorema 7.9.2 de Parlett [37], que pode ser simplificado e convenientemente escrito como [22],

$$
q_{i}^{2}=\left|\frac{P_{k-1}\left(\lambda_{i}^{k}\right)}{P_{k}^{\prime}\left(\lambda_{i}^{k}\right)}\right|,
$$

onde $P^{\prime}$, denota a derivada do polinômio característico. Trabalhando a última expressão, temos:

$$
\prod_{i=1}^{k} q_{i}^{2}=\frac{\prod_{i=1}^{k}\left|P_{k-1}\left(\lambda_{i}^{k}\right)\right|}{\prod_{i=1}^{k}\left|P_{k}^{\prime}\left(\lambda_{i}^{k}\right)\right|}
$$

o que implica,

$$
\prod_{i=1}^{k} q_{i}^{2}=\frac{\prod_{i=1}^{n-1} b_{i}^{2 i}}{\prod_{i \neq j}\left(\lambda_{i}-\lambda_{j}\right)^{2}}
$$

e conclui a prova. 


\section{Apêndice B}

\section{Elementos de Probabilidade}

\section{B.1 Introdução à Teoria da Probabilidade}

Probabilidade é a parte da matemática que estuda fenômenos, popularmente, conhecidos como incertos ou aleatórios. Esses termos se opõem a ideia de um fenômeno determinístico. De maneira simplificada, podemos entender como determinístico aquele que, sob as mesmas condições iniciais, sempre produz o mesmo resultado.

Definição 1: Ao conjunto $(\Omega)$ de todos os resultados possíveis em um fenômeno aleatório denotamos por espaço amostral.

Definição 2: Dado qualquer subconjunto $E \subset \Omega$ definimos $E$ como um evento.

Definição 3: Dizemos que dois eventos quaisquer em $\Omega$ são disjuntos, ou mutuamente exclusivos, se, e somente se, satisfazem $E \cap F=0$.

Com as definições anteriores, podemos agora formalizar o conceito de probabilidade [38].

Definição 4: Para todo evento $E \subset \Omega$ denotamos por probabilidade um número real $\mathrm{P}(E)$, o qual satisfaz as seguintes propriedades: 


$$
\begin{aligned}
& \text { I. } 0 \leq \mathrm{P}(E) \leq 1 \\
& \text { II. } \mathrm{P}(\Omega)=1 \\
& \text { III. } \mathrm{P}\left(\bigcup_{i=1}^{\infty} E_{i}\right)=\sum_{i=1}^{\infty} \mathrm{P}\left(E_{i}\right), E_{i} \cap E_{j}=0 \forall i \neq j
\end{aligned} \text {. }
$$

\section{B.2 Variáveis Aleatórias}

Um importante conceito em teoria de probabilidades é o de uma variável aleatória, que apesar de o nome levar a uma inocente confusão trata-se de fato de uma função.

Definição 1: Uma variável aleatória $X$ é uma função $X: \Omega \rightarrow \mathbb{R}$, tal que para todo $\omega \in \Omega \exists x \in \mathbb{R}$ onde $x=X(\omega)$.

Definição 2: Dado um espaço amostral $\Omega$ com probabilidades bem definidas $\mathrm{P}(\Omega)$ e considere uma variável aleatória $X$ com valores no conjunto imagem $B=X(\Omega)$. Definimos a probabilidade no conjunto imagem como, $\mathrm{P}_{X}(B)=\mathrm{P}(\{\omega \in \Omega \mid X(\omega) \in B\})$. Ao conjunto $\mathrm{P}_{X}(B)$ chamamos de distribuição de probabilidades.

Definição 3: Se o conjunto $\mathrm{P}_{X}(B)$ de valores que uma dada variável aleatória $X$ assumir for enumerável, a variável aleatória em questão é chamada de discreta. Por outro lado, se o conjunto for não enumerável a variável aleatória é chamada de contínua. Nesse caso, a probabilidade de $X$ assumir um determinado valor em um intervalo de $B_{1} \subset B$ é dado por,

$$
\int_{B_{1}} \rho_{X}(x) d x
$$


com $\rho_{X}$ conhecida como função densidade de probabilidade.

O conhecimento da função densidade de probabilidade é importante pois a partir dela é possível obter os chamados momentos de uma distribuição. Os momentos, de maneira simplificada, são parâmetros que permitem caracterizar uma determinada distribuição, e no caso contínuo, o $n$-ésimo é definido como:

$$
\left\langle X_{n}\right\rangle=\int_{-\infty}^{\infty} x^{n} \rho_{X}(x) d x
$$

Alguns momentos recebem nomes especiais, como por exemplo, o primeiro é conhecido como média de uma distribuição, ou valor esperado.

Os resultados anteriores são facilmente generalizados para as chamadas distribuições multivariadas. Dado simultaneamente $n$ variáveis aleatórias $\left\{X_{1}, \ldots, X_{n}\right\}$ em um dado espaço amostral e consideremos a probabilidade de ao mesmo tempo $\left\{X_{1}, \ldots, X_{n}\right\}$ assumir valores em conjuntos $\left\{B_{1}, \ldots, B_{n}\right\}$, respectivamente. Essas, determinam probabilidades $\mathrm{P}_{X_{1}, \ldots, X_{n}}$ em $\mathbb{R}^{n}$ através da relação,

$$
\mathrm{P}\left(\bigcap_{i=1}^{n} X_{i}^{-1}\left(B_{i}\right)\right),
$$

onde de maneira análoga ao caso contínuo de uma única variável aleatória, podemos definir uma distribuição conjunta de probabilidade, $\rho_{X_{1}, \ldots, X_{n}}\left(x_{1}, \ldots, x_{n}\right)$, pela relação,

$$
\mathrm{P}_{X_{1}, \ldots, X_{n}}(B)=\int_{B_{1}} \ldots \int_{B_{n}} \rho_{X_{1}, \ldots, X_{n}}\left(x_{1}, \ldots, x_{n}\right) \prod_{i=1}^{n} d x_{i}
$$


Definição 4: Dado um espaço amostral $\Omega$ com probabilidade $\mathrm{P}$, duas variáveis aleatórias $X_{1}$ e $X_{2}$ são ditas independentes se dois eventos $E_{1}=X_{1}^{-1}\left(B_{1}\right)$ e $E_{2}=X_{2}^{-1}\left(B_{2}\right)$ são independentes, ou seja, se qualquer escolha de $B_{1}, B_{2} \subset \mathbb{R}$ vale $\mathrm{P}\left(E_{1} \cap E_{2}\right)=\mathrm{P}\left(E_{1}\right) \cdot \mathrm{P}\left(E_{2}\right)$.

Concluindo essa discussão vale mencionar um procedimento comum, em certas circunstâncias, que consiste em aplicar uma transformação bijetora continua $\phi$ que mapeia uma variável aleatória $X$ em uma outra variável aleatória $Y$. Se $\phi$ for diferenciável podemos expressar a nova densidade de probabilidade, $g(y)$, em termos da densidade da variável original através do Jacobiano, ou seja,

$$
g(y)=\rho(x) \cdot \mid \frac{d x}{d y} \|_{x=h^{-1}(y)} .
$$

\section{B.3 Distribuições de Probabilidades}

Uma das distribuições mais conhecidas, e talvez uma das mais importantes, é a chamada distribuição normal, ou gaussiana.

Definição 1: Dizemos que uma variável aleatória $X$ possuí distribuição normal com média $\mu$ e variância $\sigma^{2}$, denotada por $N\left(\mu, \sigma^{2}\right)$, se $X$ possui função densidade dada pela expressão,

$$
\rho_{X}(x)=\frac{1}{\sqrt{2 \pi} \sigma} e^{-\left(\frac{(x-\mu)^{2}}{2 \sigma^{2}}\right)} .
$$


Em particular temos que a distribuição normal padrão, denotada por $N(0,1)$, possui função densidade igual à,

$$
\rho_{X}(x)=\frac{1}{\sqrt{2 \pi}} e^{-\left(\frac{x^{2}}{2}\right)}
$$

Uma das razões dessa distribuição ser considerada importante reside no fato do conhecido Teorema Central do Limite [38].

No contexto das matrizes tridiagonais, no ensemble $\beta$-Laguerre, a distribuição chi $\chi$, ganha papel de destaque. Dado um conjunto de variáveis aleatórias $\left\{X_{1}, \ldots, X_{n}\right\}$ independentes e identicamente distribuídas com distribuição normal padrão, definimos uma nova variável aleatória através da relação,

$$
Y=\sqrt{\sum_{i=1}^{n} X_{i}^{2}}
$$

Nessas condições, essa nova variável possui distribuição de probabilidade $\chi_{n}(y), n$ denotando o número de graus de liberdade de $Y$, e a função densidade pode ser escrita como,

$$
\chi_{n}(y)=\left\{\begin{array}{c}
\frac{x^{n-1} e^{-y^{2} / 2}}{2^{(n / 2)-1} \Gamma\left(\frac{n}{2}\right)}, y \geq 0 \\
0, \text { caso contrário }
\end{array} .\right.
$$




\section{Bibliografia}

[1] Shankar, R., Principles of quantum mechanics, 2 ed., Springer, 1994.

[2] Sakurai, J. J.. Modern Quantum Mechanics (Revised Edition). 1 : Addison Wesley, 1993.

[3] C. M. Bender and S. Boettcher, Phys. Rev. Lett. 80, 5243 (1998).

[4] A. Mostafazadeh, "Pseudo-Hermiticty versus PT-symmetry: The necessary condition for the reality of the spectrum of a non-Hermitian Hamiltonian”. J. Math. Phys. 43 (2002), pp. 205-214.

[5] Vivo, Pierpaolo ; Livan, Giacomo ; Novaes, Marcel. / Introduction to Random Matrices: Theory and Practice. Springer, 2018. (SpringerBriefs).

[6] G. Marinello,” Matrizes aleatórias no ensemble beta”, Tese de Mestrado (2014).

[7] J.J.M. Verbaarschot ;T. Wettig, Random Matrix Theory and Chiral Symmetry in QCD, Annual Review of Nuclear and Particle Science 2000 50:1, 343-410

[8] C.W.J. Beenakker, Rev. Mod. Phys. 69 (1997) 731.

[9] A.F. Andreev, Zh. Eksp. Teor. Fiz. 46 (1964) 1823.

[10] D. Fox and P.B. Kahn, Phys. Rev. 134 (1964) B1151.

[11] Goulart, C.A.; Pato, M.P. Entanglement of Pseudo-Hermitian Random States. Entropy 2020, 22, 1109. 
[12] J. Wishart, Generalized product moment distribution in samples, Biometrika 20A.1-2 (1928), pp. $32-52$

[13] I. Dumitriu ; A. Edelman. Matrix models for beta ensembles. J. Math. Phy., 43(11):5830-5847, 2002.

[14] Forrester, P.J.: Log-Gases and Random Matrices. Princeton University Press, Princeton (2010).

[15] W. Van Assche, Asymptotics for orthogonal polynomials (Springer, 2006)

[16] L.M. Mehta, Randon Matrices, Academic Press, 2004.

[17] R. J. Muirhead, Aspects of Multivariate Statiscal Theory, Wiley, 1982.

[18] Y. V. Fyodorov, Introduction to the random matrix theory: Gaussian Unitary Ensemble and beyond. In F. Mezzadri \& N. Snaith (Eds.), Recent Perspectives in Random Matrix Theory and Number Theory (London Mathematical Society Lecture Note Series, pp. 31-78), 2004.

[19] Kornyik, Miklós \& Michaletzky, G.. (2016). On the moments of roots of Laguerre-polynomials and the Marchenko-Pastur law.

[20] Bohigas, O ; Pato, Mauricio., Decomposition of spectral density in individual eigenvalue contributions. Journal of Physics A: Mathematical and Theoretical. 43. (2010).

[21] Marinello, G. \& Pato, Mauricio., Random non-Hermitian tight-binding models. Journal of Physics: Conference Series. 738. (2016).

[22] I. Dumitriu, "Eigenvalues Statistics for Beta-Ensembles". Tese de doutoramento. Massachusetts Institute of Technology, 2003. 
[23] G. H. Golub and C. Reinsch, "Singular value decomposition and least squares solutions," Numer. Math., vol. 14, pp. 403-420, 1970.

[24] A. Householder. Unitary triangularization of a nonsymmetric matrix. Journal of the ACM (JACM), Association for Computing Machinery, 1958.

[25] Wilkinson, J.H. Householder's method for symmetric matrices. Numer. Math. 4, 354-361 (1962).

[26] Bender, Carl \& Brody, Dorje \& Jones, Hugh. Complex Extension of Quantum Mechanics. Physical review letters. 89 (2003).

[27] A. Mostafazadeh. PSEUDO-HERMITIAN REPRESENTATION OF QUANTUM MECHANICS. International Journal of Geometric Methods in Modern Physics. 07 (2012).

[28] Hossein Mehri-Dehnavi et al 2010 J. Phys. A: Math. Theor. 43145301

[29] Bohigas, O.; Pato, M.P. Non-Hermitian $\beta$-ensemble with real eigenvalues. AIP Adv. 2013, 3, 032130 .

[30] Marinello, G. \& Pato, Mauricio. Pseudo-Hermitian anti-Hermitian ensemble of Gaussian matrices. Physical Review E. 96 (2017).

[31] Sherif M. Abuelenin, Adel Y. Abul-Magd,Effect of Unfolding on the Spectral Statistics of Adjacency Matrices of Complex Networks,Procedia Computer Science,Vol. 12,2012,pp. 69-74.

[32] Romuald A Janik and Maciej A Nowak 2003 J. Phys. A: Math. Gen. 363629

[33] E. P. Wigner. "Characteristics Vectors of Bordered Matrices with Infinite Dimensions II." Annals of Mathematics, vol. 65, no. 2, 1957, pp. 203-207. 
[34] E. L. Lima, “Álgebra Linear”, IMPA, 2018.

[35] R. A. Horn; C. R. Johnson. Matrix Analysis, 2 ed., Cambridge Press, 2012.

[36] Ford, William. Numerical Linear Algebra with Applications using MATLAB (2014).

[37] B. N. Parlett. The Symmetric Eigenvalue Problem. Philadelphia: Society for Industrial and Applied Mathematics (1998).

[38] S. Ross, Probabilidade: Um curso moderno com aplicações, Bookman, 2010. 